\title{
Bifurcation of Limit Cycles by Perturbing a Piecewise Linear Hamiltonian System
}

\author{
Yanqin Xiong and Maoan Han \\ Department of Mathematics, Shanghai Normal University, Shanghai 200234, China \\ Correspondence should be addressed to Maoan Han; mahan@shnu.edu.cn \\ Received 5 October 2012; Accepted 21 November 2012 \\ Academic Editor: Yonghui Xia
}

Copyright (C) 2013 Y. Xiong and M. Han. This is an open access article distributed under the Creative Commons Attribution License, which permits unrestricted use, distribution, and reproduction in any medium, provided the original work is properly cited.

This paper concerns limit cycle bifurcations by perturbing a piecewise linear Hamiltonian system. We first obtain all phase portraits of the unperturbed system having at least one family of periodic orbits. By using the first-order Melnikov function of the piecewise near-Hamiltonian system, we investigate the maximal number of limit cycles that bifurcate from a global center up to first order of $\varepsilon$.

\section{Introduction and Main Results}

Recently, piecewise smooth dynamical systems have been well concerned, especially in the scientific problems and engineering applications. For example, see the works of Filippov [1], Kunze [2], di Bernardo et al. [3], and the references therein. Because of the variety of the nonsmoothness, there can appear many complicated phenomena in piecewise smooth dynamical systems such as stability (see $[4,5]$ ), chaos (see [6]), and limit cycle bifurcation (see [7-10]). Here, we are more concerned with bifurcation of limit cycles in a perturbed piecewise linear Hamiltonian system:

$$
\begin{gathered}
\dot{x}=y+\varepsilon p(x, y, \delta), \\
\dot{y}=-g(x)+\varepsilon q(x, y, \delta),
\end{gathered}
$$

where $\varepsilon>0$ is a sufficiently small real parameter,

$$
g(x)= \begin{cases}a_{1} x+a_{0}, & x \geq 0 \\ b_{1} x+b_{0}, & x<0\end{cases}
$$

with $a_{1}, a_{0}, b_{1}$, and $b_{0}$ real numbers satisfying $a_{1}^{2}+a_{0}^{2} \neq 0, b_{1}^{2}+$ $b_{0}^{2} \neq 0$,

$$
\begin{aligned}
& p(x, y, \delta)= \begin{cases}p^{+}(x, y, \delta)=\sum_{i+j=0}^{n} a_{i j}^{+} x^{i} y^{j}, & x \geq 0, \\
p^{-}(x, y, \delta)=\sum_{i+j=0}^{n} a_{i j}^{-} x^{i} y^{j}, & x<0,\end{cases} \\
& q(x, y, \delta)= \begin{cases}q^{+}(x, y, \delta)=\sum_{i+j=0}^{n} b_{i j}^{+} x^{i} y^{j}, & x \geq 0, \\
q^{-}(x, y, \delta)=\sum_{i+j=0}^{n} b_{i j}^{-} x^{i} y^{j}, & x<0,\end{cases}
\end{aligned}
$$

and $\delta=\left(a_{i j}^{+}, a_{i j}^{-}, b_{i j}^{+}, b_{i j}^{-}\right) \in D \subset \mathbb{R}^{2(n+1)(n+2)}$ with $D$ compact. Then system (1) has two subsystems

$$
\dot{x}=y+\varepsilon p^{+}(x, y, \delta)
$$

$$
\begin{gathered}
\dot{y}=-a_{1} x-a_{0}+\varepsilon q^{+}(x, y, \delta), \\
\dot{x}=y+\varepsilon p^{-}(x, y, \delta), \\
\dot{y}=-b_{1} x-b_{0}+\varepsilon q^{-}(x, y, \delta),
\end{gathered}
$$


which are called the right subsystem and the left subsystem, respectively. For $\varepsilon=0$, systems (5a) and (5b) are Hamiltonian with the Hamiltonian functions, respectively,

$$
\begin{aligned}
& H^{+}(x, y)=\frac{1}{2} y^{2}+\frac{1}{2} a_{1} x^{2}+a_{0} x, \\
& H^{-}(x, y)=\frac{1}{2} y^{2}+\frac{1}{2} b_{1} x^{2}+b_{0} x .
\end{aligned}
$$

Note that the phase portrait of the linear system

$$
\begin{gathered}
\dot{x}=y, \\
\dot{y}=-a x-b,
\end{gathered}
$$

with $a^{2}+b^{2} \neq 0$ has possibly the following four different phase portraits on the plane (see Figure 1).

Then, one can find that system (1) $\left.\right|_{\varepsilon=0}$ can have 13 different phase portraits (see Figure 2) when at least one family of periodic orbits appears.

We remark that in Figure 2,

GC: global center,

Ho: homoclinic,

He: heteroclinic,

$C^{+}$: center in the region $\{(x, y) \mid x>0\}$,

$C^{-}$: center in the region $\{(x, y) \mid x<0\}$,

$S^{+}$: saddle in the region $\{(x, y) \mid x>0\}$,

$S^{-}$: saddle in the region $\{(x, y) \mid x<0\}$,

$L^{+}$: curvilinear or straightline in the region $\{(x, y) \mid$ $x>0\}$,

$L^{-}$: curvilinear or straightline in the region $\{(x, y) \mid$ $x<0\}$.

It is easy to obtain the following Table 1 which shows conditions for each possible phase portrait appearing above. Also, cases (3), (5), (7), (9), and (13) in Figure 2 are equivalent to cases $(2),(6),(8),(10)$, and (12), respectively, by making the transformation

$$
(x, y) \longrightarrow(-x, y)
$$

together with time rescaling $d t=-d \tau$.

The authors Liu and Han [7] studied system (1) in a subcase of the case (1) of Figure 2 by taking $a_{1}=b_{1}=1, a_{0}=$ $b_{0}=0$. By using the first order Melnikov function, they proved that the maximal number of limit cycles on Poincaré bifurcations is $\mathrm{n}$ up to first-order in $\varepsilon$. The authors Liang et al. [8] considered system (1) in the case (5) of Figure 2 by taking $a_{1}=-1, a_{0}=1, b_{1}=1$, and $b_{0}=0$. By using the same method, they gave lower bounds of the maximal number of limit cycles in Hopf, and Homoclinic bifurcations, and derived an upper bound of the maximal number of limit cycles bifurcating from the periodic annulus between the center and the Homoclinic loop up to the first-order in $\varepsilon$. Clearly, the maximal number of limit cycles in the case (7) or (8) of Figure 2 is [ $(n-1) / 2]$ on Poincaré, Hopf and Homoclinic bifurcations up to first-order in $\varepsilon$, by using the first order Melnikov function.

This paper focuses on studying the limit cycle bifurcations of system (1) in the case (1) of Figure 2 by using the first order Melnikov function. That is, system (1) satisfies

$$
\begin{array}{ccc}
a_{1} \geq 0, & a_{0} \geq 0, & a_{0}+a_{1}>0, \\
b_{1} \geq 0, & b_{0} \leq 0, & b_{0}<b_{1} .
\end{array}
$$

Clearly, system (1) $\left.\right|_{\varepsilon=0}$ satisfying (9) has a family of periodic orbits

$$
\begin{aligned}
L_{h}= & L_{h}^{+} \cup L_{h}^{-} \\
= & \left\{(x, y) \mid H^{+}(x, y)=h\right\} \\
& \cup\left\{(x, y) \mid H^{-}(x, y)=h\right\}, \quad h>0,
\end{aligned}
$$

such that the limit of $L_{h}$ as $h \rightarrow 0^{+}$is the origin. The intersection points of the closed curve $L_{h}$ with the positive $y$ axis and the negative $y$-axis are denoted by $A(h)=(0, \sqrt{2 h})$ and $A_{1}(h)=(0,-\sqrt{2 h})$, respectively. Let

$$
\begin{gathered}
M^{+}(h, \delta)=\int_{\widehat{A A_{1}}} q^{+} d x-p^{+} d y, \\
M^{-}(h, \delta)=\int_{\widehat{A_{1} A}} q^{-} d x-p^{-} d y, \quad h>0 .
\end{gathered}
$$

Then, from Liu and Han [7], the first-order Melnikove function corresponding to system (1) is

$$
M(h, \delta)=M^{+}(h, \delta)+M^{-}(h, \delta), \quad h \in(0,+\infty) .
$$

Let $Z(n)$ denote the maximal number of zeros of $M(h, \delta)$ for $h>0$ and $N(n)$ the cyclicity of system (1) at the origin. Then, we can obtain the following.

Theorem 1. Let (9) be satisfied. For any given $n \geq 1$, one has Table 2.

This paper is organized as follows. In Section 2, we will provide some preliminary lemmas, which will be used to prove the main results. In Section 3, we present the proof of Theorem 1.

\section{Preliminary Lemmas}

In this section, we will derive expressions of $M^{+}(h, \delta)$, $M^{-}(h, \delta)$ in (11). First, we have the following.

Lemma 2. Suppose system (1) satisfies (9). Then,

$$
\text { (i) } M^{+}(h, \delta) \text { in (11) can be written as }
$$

$$
M^{+}(h, \delta)=M_{1}^{+}(h, \delta)+\sum_{2 k=0}^{n} \frac{2^{k+1+1 / 2}}{2 k+1} a_{0,2 k}^{+} h^{k+1 / 2},
$$

where

$$
M_{1}^{+}(h, \delta)=\sum_{i+j=0}^{n-1} \bar{p}_{i j}^{+} \int_{\widehat{A_{1} A}} x^{i+1} y^{j} d y=\sum_{i+j=0}^{n-1} \bar{q}_{i j}^{+} \int_{\widehat{A_{1} A}} x^{i} y^{j+1} d x
$$


TABLE 1: Coefficient conditions for phase portraits (1)-(13).

\begin{tabular}{lcccc}
\hline Coefficient conditions & $\begin{array}{c}a_{1} \geq 0, a_{0} \geq 0 \\
a_{1}+a_{0}>0\end{array}$ & $\begin{array}{c}a_{1}>0 \\
a_{0}<0\end{array}$ & $\begin{array}{c}a_{1} \leq 0, a_{0} \leq 0 \\
a_{1}+a_{0}<0\end{array}$ & $\begin{array}{c}a_{1}<0 \\
a_{0}>0\end{array}$ \\
\hline$b_{1} \geq 0, b_{0} \leq 0, b_{1}>b_{0}$ & $(1)$ & $(2)$ & & $(5)$ \\
$b_{1}>0, b_{0}>0$ & $(3)$ & $(4)$ & $(7)$ & $(9)$ \\
$b_{1} \leq 0, b_{0} \geq 0, b_{0}>b_{1}$ & & & $(11)\left(a_{0}^{2} b_{1}=a_{1} b_{0}^{2}\right)$, \\
& & $(10)$ & $(12)\left(a_{0}^{2} b_{1}>a_{1} b_{0}^{2}\right)$, \\
$b_{1}<0, b_{0}<0$ & $(6)$ & & $(13)\left(a_{0}^{2} b_{1}<a_{1} b_{0}^{2}\right)$ \\
\hline
\end{tabular}

TABLE 2

\begin{tabular}{lccc}
\hline & $a_{1}>0, a_{0}=0$ & $a_{1}=0, a_{0}>0$ & $a_{1}>0, a_{0}>0$ \\
\hline$b_{1}>0$, & $Z(n)=n$, & $Z(n)=n+\left[\frac{n+1}{2}\right]$, & $Z(n)=n+\left[\frac{n+1}{2}\right]$, \\
$b_{0}=0$ & $N(n) \geq n$ & $N(n) \geq n+\left[\frac{n+1}{2}\right]$ & $N(n) \geq n+\left[\frac{n+1}{2}\right]$
\end{tabular}

\begin{tabular}{|c|c|c|c|c|c|c|}
\hline$b_{1}=0$ & $Z(n)=n+\left[\frac{n+1}{2}\right]$ & $Z(n)=n$ & $n+$ & $\frac{n+1}{2}$ & $\leq Z(n) \leq n+2$ & {$\left[\frac{n+1}{2}\right.$} \\
\hline$b_{0}<0$ & $N(n) \geq n+\left[\frac{n+1}{2}\right]$ & $N(n) \geq n$ & & & n) $\geq n+\left[\frac{n+1}{2}\right]$, & \\
\hline
\end{tabular}

\begin{tabular}{|c|c|c|c|c|c|c|c|}
\hline$b_{1}>0$, & $Z(n)=n+$ & {$\left[\frac{n+1}{2}\right]$} & $n+\left[\frac{n+1}{2}\right] \leq Z(n) \leq n+2$ & {$\left[\frac{n+1}{2}\right.$} & $n \leq Z(n) \leq n+$ & {$\left[\frac{n+1}{2}\right]$} &,$\frac{a_{0}^{2}}{a_{1}}=\frac{b_{0}^{2}}{b_{1}}$ \\
\hline$b_{0}<0$ & $N(n) \geq n+$ & {$\left[\frac{n+1}{2}\right]$} & $N(n) \geq n+\left[\frac{n+1}{2}\right]$ & & $n \leq Z(n) \leq n+2$ & {$\left[\frac{n+1}{2}\right.$} & ],$\frac{a_{0}^{2}}{a_{1}} \neq$ \\
\hline
\end{tabular}

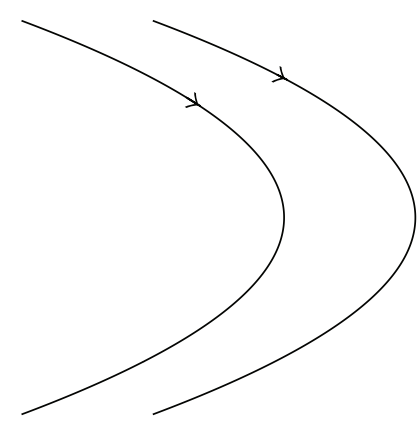

(a) $a=0, b>0$

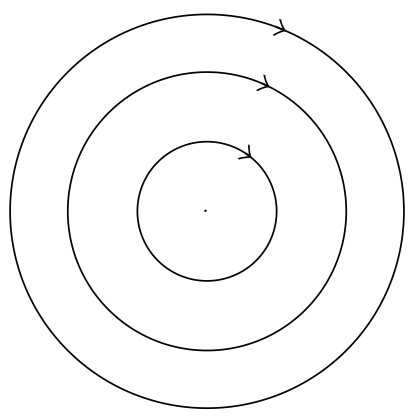

(c) $a>0$

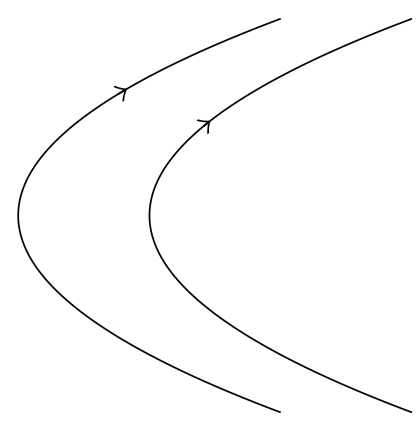

(b) $a=0, b<0$

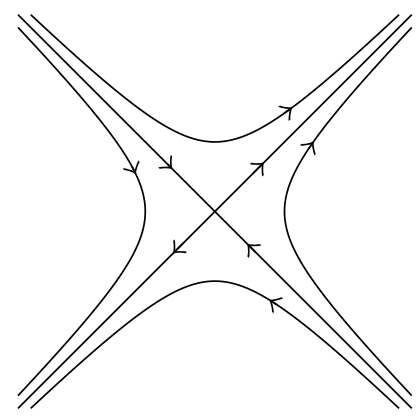

(d) $a<0$

Figure 1: The possible phase portraits of system (7). 


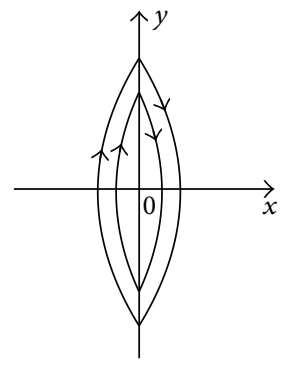

(1)

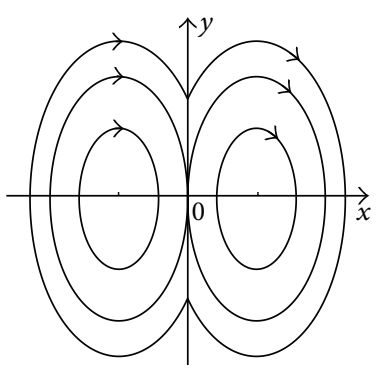

(4)

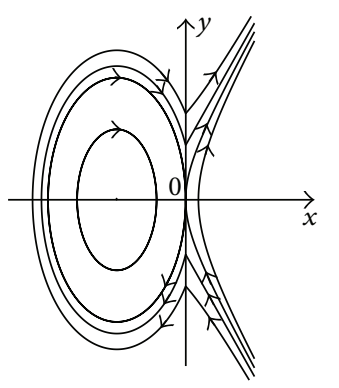

(7)

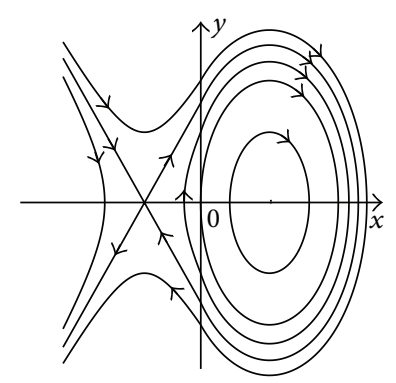

(10)

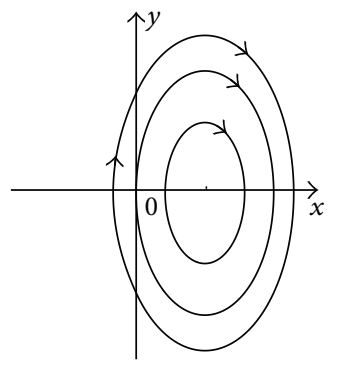

(2)

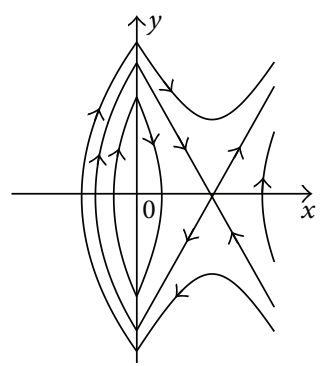

(5)

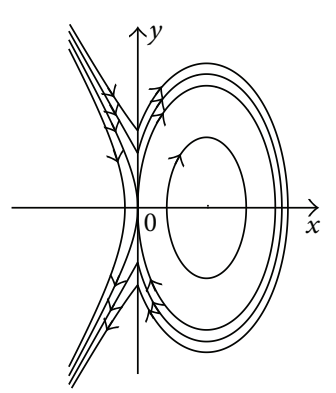

(8)

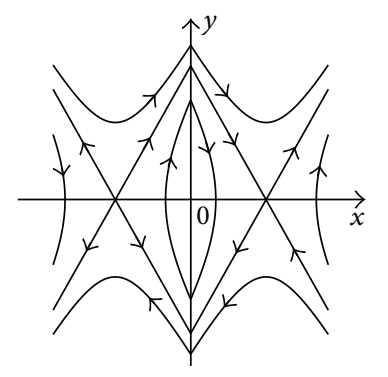

(11)

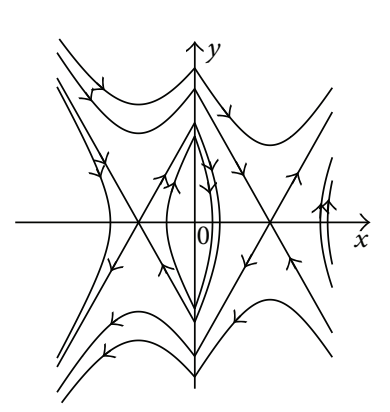

(13)

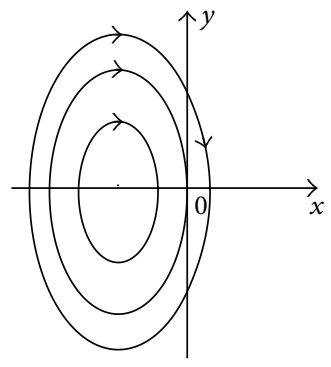

(3)

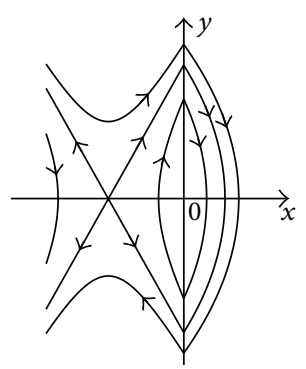

(6)

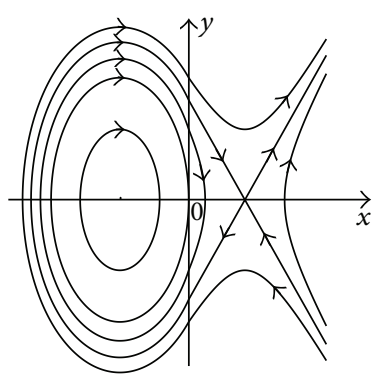

(9)

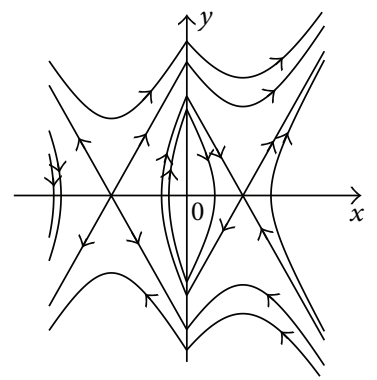

(12) 
with

$$
\bar{p}_{i j}^{+}=a_{i+1, j}^{+}+\frac{j+1}{i+1} b_{i, j+1}^{+}, \quad \bar{q}_{i j}^{+}=b_{i, j+1}^{+}+\frac{i+1}{j+1} a_{i+1, j}^{+} .
$$

(ii) $(h, \delta)$ in (11) can be expressed as

$$
\begin{gathered}
M^{-}(h, \delta)=M_{1}^{-}(h, \delta)-\sum_{2 k=0}^{n} \frac{2^{k+1+1 / 2}}{2 k+1} a_{0,2 k}^{-} h^{k+1 / 2}, \\
\text { where } \\
M_{1}^{-}(h, \delta)=\sum_{i+j=0}^{n-1} \bar{p}_{i j}^{-} \int_{\widehat{A_{1} A}} x^{i+1} y^{j} d y=\sum_{i+j=0}^{n-1} \bar{q}_{i j}^{-} \int_{\widehat{A_{1} A}} x^{i} y^{j+1} d x, \\
\text { with } \quad \bar{q}_{i j}^{-}=b_{i, j+1}^{-}+\frac{i+1}{j+1} a_{i+1, j}^{-} .
\end{gathered}
$$

Proof. We only prove (i) since (ii) can be verified in a similar way. By (11), we obtain

$$
\begin{aligned}
M^{+}(h, \delta)= & \int_{\widehat{A A_{1}}} q^{+}(x, y, \delta) d x-p^{+}(x, y, \delta) d y \\
& +\int_{\overrightarrow{A_{1} A}} q^{+}(x, y, \delta) d x-p^{+}(x, y, \delta) d y \\
& -\int_{\overrightarrow{A_{1} A}} q^{+}(x, y, \delta) d x-p^{+}(x, y, \delta) d y \\
= & \oint_{\widehat{A A_{1} \cup A_{1} A}} q^{+}(x, y, \delta) d x-p^{+}(x, y, \delta) d y \\
& +\int_{\overrightarrow{A_{1} A}} p^{+}(0, y, \delta) d y,
\end{aligned}
$$

which follows that by Green formula and (3)

$$
M^{+}(h, \delta)=M_{1}^{+}(h, \delta)+\sum_{2 k=0}^{n} \frac{2^{k+1+1 / 2}}{2 k+1} a_{0,2 k}^{+} h^{k+1 / 2},
$$

where

$$
M_{1}^{+}(h, \delta)=\iint_{\text {int } \widehat{A A_{1}} \cup \overrightarrow{A_{1} A}}\left(p_{x}^{+}+q_{y}^{+}\right) d x d y .
$$

Then, by Green formula again

$$
\begin{aligned}
M_{1}^{+}(h, \delta) & =-\oint_{\widehat{A A_{1} \cup \overrightarrow{A_{1} A}}} \vec{p}^{+}(x, y, \delta) d y \\
& =\oint_{\widehat{A A_{1}} \cup \overrightarrow{A_{1} A}} \bar{q}^{+}(x, y, \delta) d x,
\end{aligned}
$$

where

$$
\begin{aligned}
& \bar{p}^{+}(x, y, \delta)=p^{+}(x, y, \delta)-p^{+}(0, y, \delta)+\int_{0}^{x} q_{y}^{+}(u, y, \delta) d u \\
& \bar{q}^{+}(x, y, \delta)=q^{+}(x, y, \delta)-q^{+}(x, 0, \delta)+\int_{0}^{y} p_{x}^{+}(x, v, \delta) d v
\end{aligned}
$$

By (3), (4), and the above formulas, we have

$$
\begin{aligned}
\bar{p}^{+}(x, y, \delta) & =\sum_{i+j=0}^{n} a_{i j}^{+} x^{i} y^{j}-\sum_{j=0}^{n} a_{0 j}^{+} y^{j}+\sum_{i+j=1}^{n} \frac{j}{i+1} b_{i j}^{+} x^{i+1} y^{j-1} \\
& =x \sum_{i+j=0}^{n-1}\left(a_{i+1, j}^{+}+\frac{j+1}{i+1} b_{i, j+1}^{+}\right) x^{i} y^{j} \\
& =x \sum_{i+j=0}^{n-1} \bar{p}_{i j}^{+} x^{i} y^{j}
\end{aligned}
$$$$
\bar{q}^{+}(x, y, \delta)=\sum_{i+j=0}^{n} b_{i j}^{+} x^{i} y^{j}-\sum_{j=0}^{n} b_{i 0}^{+} x^{i}+\sum_{i+j=1}^{n} \frac{i}{j+1} a_{i j}^{+} x^{i-1} y^{j+1}
$$$$
=y \sum_{i+j=0}^{n-1}\left(b_{i, j+1}^{+}+\frac{i+1}{j+1} a_{i+1, j}^{+}\right) x^{i} y^{j}
$$$$
=y \sum_{i+j=0}^{n-1} \bar{q}_{i j}^{+} x^{i} y^{j}
$$

Combining (20)-(25) gives (13) and (14). Thus, the proof is ended.

Then, using Lemma 2 and (6) we can obtain the following three lemmas.

Lemma 3. (i) If $a_{1}=0, a_{0}>0$, then $M^{+}(h, \delta)$ in (11) has form

$$
M^{+}(h, \delta)=h^{1 / 2} \sum_{i+2 k=0}^{n} B_{i, 2 k}^{+} h^{i+k}
$$

where

$$
\begin{aligned}
B_{0,2 k}^{+}= & \frac{2^{k+1+1 / 2} a_{0,2 k}^{+}}{2 k+1}, \\
B_{i, 2 k}^{+}= & \frac{2^{k+1+1 / 2}}{a_{0}^{i}}\left(a_{i, 2 k}^{+}+\frac{2 k+1}{i} b_{i-1,2 k+1}^{+}\right) \\
& \times \int_{0}^{\pi / 2} \sin ^{2 k} \theta \cos ^{i+1} \theta d \theta, \quad 1 \leq i \leq n .
\end{aligned}
$$

(ii) If $b_{1}=0, b_{0}<0$, then we have

$$
M^{-}(h, \delta)=h^{1 / 2} \sum_{i+2 k=0}^{n} B_{i, 2 k}^{-} h^{i+k}
$$


where

$$
\begin{aligned}
B_{0,2 k}^{-}= & -\frac{2^{k+1+1 / 2} a_{0,2 k}^{-}}{2 k+1}, \\
B_{i, 2 k}^{-}= & \frac{-2^{k+1+1 / 2}}{b_{0}^{i}}\left(a_{i, 2 k}^{-}+\frac{2 k+1}{i} b_{i-1,2 k+1}^{-}\right) \\
& \times \int_{0}^{\pi / 2} \sin ^{2 k} \theta \cos ^{2 i+1} \theta d \theta, \quad 1 \leq i \leq n .
\end{aligned}
$$

Proof. Note that along $\widehat{\mathrm{AA}_{1}}, x=h / a_{0}-\left(1 / 2 a_{0}\right) y^{2}$. Then, inserting it into (14) follows that

$$
\begin{aligned}
M_{1}^{+}(h, \delta) & =\sum_{i+j=0}^{n-1} \bar{p}_{i j}^{+} \int_{-\sqrt{2 h}}^{\sqrt{2 h}}\left(\frac{h}{a_{0}}-\frac{1}{2 a_{0}} y^{2}\right)^{i+1} y^{j} d y \\
& =\sum_{i+2 k=0}^{n-1} \frac{\bar{p}_{i, 2 k}^{+}}{2^{i} a_{0}^{i+1}} \int_{0}^{\sqrt{2 h}}\left(2 h-y^{2}\right)^{i+1} y^{2 k} d y .
\end{aligned}
$$

Let $y=\sqrt{2 h} \sin \theta$. Then we have $d y=\sqrt{2 h} \cos \theta d \theta$ and the above integral can be carried into

$$
\begin{aligned}
\int_{0}^{\sqrt{2 h}}\left(2 h-y^{2}\right)^{i+1} y^{2 k} d y= & (2 h)^{i+1+k+(1 / 2)} \\
& \times \int_{0}^{\pi / 2} \sin ^{2 k} \theta \cos ^{2(i+1)+1} \theta \mathrm{d} \theta .
\end{aligned}
$$

Thus, using (30) and the above equation we can write (13) as

$$
\begin{aligned}
M^{+}(h, \delta)= & \sum_{i+2 k=0}^{n-1} \frac{2^{k+1+1 / 2} \bar{p}_{i, 2 k}^{+}}{a_{0}^{i+1}} \\
& \times \int_{0}^{\pi / 2} \sin ^{2 k} \theta \cos ^{2(i+1)+1} \theta d \theta \times h^{i+1+k+1 / 2} \\
& +\sum_{2 k=0}^{n} \frac{2^{k+1+1 / 2} a_{0,2 k}^{+}}{2 k+1} h^{k+1 / 2} \\
= & h^{1 / 2} \sum_{i+2 k=0}^{n} B_{i, 2 k}^{+} h^{i+k}
\end{aligned}
$$

where

$$
\begin{aligned}
B_{0,2 k}^{+} & =\frac{2^{k+1+1 / 2} a_{0,2 k}^{+}}{2 k+1}, \\
B_{i, 2 k}^{+} & =\frac{2^{k+1+1 / 2} \bar{p}_{i-1,2 k}^{+}}{a_{0}^{i}} \int_{0}^{\pi / 2} \sin ^{2 k} \theta \cos ^{2 i+1} \theta d \theta, \quad 1 \leq i \leq n,
\end{aligned}
$$

which gives (i) by (15). Thus, (i) holds and we can prove (ii) in the same way by (16)-(18). This ends the proof.
Lemma 4. Let system (5a) satisfy (3) and (4). Then

(i) If $a_{1}>0, a_{0}=0, M^{+}(h, \delta)$ has the expression

$$
M^{+}(h, \delta)=\sqrt{h} \sum_{i+2 k=0}^{n} A_{i, 2 k}^{+}(\sqrt{h})^{i+2 k},
$$

where

$$
\begin{aligned}
A_{0,2 k}^{+}= & \frac{2(\sqrt{2})^{2 k+1} a_{0,2 k}^{+}}{2 k+1}, \\
A_{i, 2 k}^{+}= & \frac{2(\sqrt{2})^{2 k+1+i}}{\left(\sqrt{a_{1}}\right)^{i}}\left(b_{i-1,2 k+1}^{+}+\frac{i}{2 k+1} a_{i, 2 k}^{+}\right) \\
& \times \int_{0}^{\pi / 2} \sin ^{i-1} \theta \cos ^{2 k+2} \theta d \theta, \quad i \geq 1 .
\end{aligned}
$$

(ii) If $a_{1}>0, a_{0} \neq 0, M^{+}(h, \delta)$ can be written as

$$
\begin{aligned}
M^{+}(h, \delta)= & \sqrt{h}\left[\sum_{i+2 k=0}^{n-1}\left(b_{i, 2 k+1}^{+}+\frac{i+1}{2 k+1} a_{i+1,2 k}^{+}\right) \phi_{i k}^{+}(h)\right. \\
& \left.+\sum_{2 k=0}^{n} \frac{2^{k+1+1 / 2} a_{0,2 k}^{+}}{2 k+1} h^{k}\right] \\
& +\sum_{i+2 k=0}^{n-1}\left(b_{i, 2 k+1}^{+}+\frac{i+1}{2 k+1} a_{i+1,2 k}^{+}\right) \\
& \times \sum_{r=0, \text { reven }}^{i} \alpha_{i r k}^{+}\left(2 h+\frac{a_{0}^{2}}{a_{1}}\right)^{k+r / 2} \bar{I}_{00}^{+}(h, \delta),
\end{aligned}
$$

or

$$
\begin{aligned}
M^{+}(h, \delta)= & \sqrt{h} \psi_{[n / 2]}^{+}(h, \delta) \\
& +\left(2 h+\frac{a_{0}^{2}}{a_{1}}\right) \varphi_{[(n-1) / 2]}^{+}\left(2 h+\frac{a_{0}^{2}}{a_{1}}, \delta\right) \\
& \times\left(\frac{\pi}{2}-\arcsin \frac{a_{0}}{\sqrt{2 a_{1} h+a_{0}^{2}}}\right),
\end{aligned}
$$

where

$$
\bar{I}_{00}^{+}(h, \delta)=\int_{a_{0} / \sqrt{a_{1}}}^{\sqrt{2 h+a_{0}^{2} / a_{1}}} \sqrt{2 h+\frac{a_{0}^{2}}{a_{1}}-v^{2}} d v,
$$

each $\alpha_{i r k}^{+}$is a nonzero constant and $\phi_{i k}^{+}, \psi_{[n / 2]}^{+}, \varphi_{[(n-1) / 2]}^{+}$ are polynomials of degree $k+[(i+1) / 2],[n / 2],[(n-$ 1)/2], respectively. 
Proof. Since $y= \pm \sqrt{2 h-a_{1} x^{2}-2 a_{0} x}$ along the curve $\widehat{A A_{1}}, M_{1}^{+}(h, \delta)$ in (14) becomes

$$
\begin{aligned}
& M_{1}^{+}(h, \delta) \\
& =\sum_{i+j=0}^{n-1} \bar{q}_{i j}^{+} \int_{\widehat{A A_{1}}} x^{i} y^{j+1} d x \\
& =\sum_{i+j=0}^{n-1} \bar{q}_{i j}^{+}\left[\int_{0}^{\left(-a_{0}+\sqrt{2 a_{1} h+a_{0}^{2}}\right) / a_{1}} x^{i}\left(2 h-a_{1} x^{2}-2 a_{0} x\right)^{(j+1) / 2} d x\right] \\
& +\int_{\left(-a_{0}+\sqrt{2 a_{1} h+a_{0}^{2}}\right) / a_{1}}^{0} x^{i}(-1)^{j+1}\left(2 h-a_{1} x^{2}-2 a_{0} x\right)^{(j+1) / 2} d x \\
& =\sum_{i+2 k=0}^{n-1} 2 \bar{q}_{i, 2 k}^{+} I_{i, 2 k}^{+}(h, \delta),
\end{aligned}
$$

where

$$
\begin{aligned}
& I_{i, 2 k}^{+}(h, \delta) \\
& =\int_{0}^{\left(-a_{0}+\sqrt{2 a_{1} h+a_{0}^{2}}\right) / a_{1}} x^{i}\left[2 h+\frac{a_{0}^{2}}{a_{1}}-a_{1}\left(x+\frac{a_{0}}{a_{1}}\right)^{2}\right]^{k+1 / 2} d x .
\end{aligned}
$$

Let $v=\sqrt{a_{1}}\left(x+a_{0} / a_{1}\right)$. Then, we have $d v=\sqrt{a_{1}} d x$ and the above equation becomes

$$
\begin{aligned}
I_{i, 2 k}^{+}(h, \delta)= & \frac{1}{\left(\sqrt{a_{1}}\right)^{i+1}} \int_{a_{0} / \sqrt{a_{1}}}^{\sqrt{2 h+a_{0}^{2} / a_{1}}}\left(v-\frac{a_{0}}{\sqrt{a_{1}}}\right)^{i} \\
& \times\left(2 h+\frac{a_{0}^{2}}{a_{1}}-v^{2}\right)^{k+(1 / 2)} d v .
\end{aligned}
$$

For $a_{0}=0$, make the transformation $v=\sqrt{2 h} \sin \theta$. Then, we have by (41)

$$
I_{i, 2 k}^{+}(h, \delta)=\frac{(\sqrt{2 h})^{2 k+1+i+1}}{\left(\sqrt{a_{1}}\right)^{i+1}} \int_{0}^{\pi / 2} \sin ^{i} \theta \cos ^{2 k+2} \theta d \theta .
$$

Substituting the above formula into (37), together with (13), gives that

$$
\begin{aligned}
M^{+}(h, \delta)= & \sum_{i+2 k=0}^{n-1} \frac{2 \bar{q}_{i, 2 k}^{+}}{\left(\sqrt{a_{1}}\right)^{i+1}} \\
& \times \int_{0}^{\pi / 2} \sin ^{i} \theta \cos ^{2 k+2} \theta d \theta \times(\sqrt{2 h})^{2 k+1+i+1} \\
& +\sum_{2 k=0}^{n} \frac{2 a_{0,2 k}^{+}}{2 k+1}(\sqrt{2 h})^{2 k+1} \\
= & \sum_{i+2 k=0}^{n} A_{i, 2 k}^{+}(\sqrt{h})^{2 k+1+i}
\end{aligned}
$$

where

$$
\begin{aligned}
A_{0,2 k}^{+}= & \frac{2(\sqrt{2})^{2 k+1} a_{0,2 k}^{+}}{2 k+1}, \\
A_{i, 2 k}^{+}= & \frac{2(\sqrt{2})^{2 k+1+i} \bar{q}_{i-1,2 k}^{+}}{\left(\sqrt{a_{1}}\right)^{i}} \\
& \times \int_{0}^{\pi / 2} \sin ^{i-1} \theta \cos ^{2 k+2} \theta d \theta, \quad 1 \leq i \leq n .
\end{aligned}
$$

Thus, by (15) and the above discussion we know that (i) holds. For $a_{0} \neq 0$, (41) can be represented as

$$
I_{i, 2 k}^{+}(h, \delta)=\frac{1}{\left(\sqrt{a_{1}}\right)^{i+1}} \sum_{r=0}^{i} C_{i}^{r}\left(-\frac{a_{0}}{\sqrt{a_{1}}}\right)^{i-r} \bar{I}_{r k}^{+}(h, \delta),
$$

where

$$
\bar{I}_{r k}^{+}(h, \delta)=\int_{a_{0} / \sqrt{a_{1}}}^{\sqrt{2 h+a_{0}^{2} / a_{1}}} v^{r}\left(2 h+\frac{a_{0}^{2}}{a_{1}}-v^{2}\right)^{k+1 / 2} d v .
$$

Recall that

$$
\begin{gathered}
\int v^{r}\left(2 h+\frac{a_{0}^{2}}{a_{1}}-v^{2}\right)^{k+1 / 2} d v \\
=\frac{v^{r+1}\left(2 h+a_{0}^{2} / a_{1}-v^{2}\right)^{k+1 / 2}}{2 k+2+r} \\
+\frac{(2 k+1)\left(2 h+a_{0}^{2} / a_{1}\right)}{2 k+2+r} \\
\quad \times \int v^{r}\left(2 h+\frac{a_{0}^{2}}{a_{1}}-v^{2}\right)^{k-1 / 2} d v .
\end{gathered}
$$

Then, by (46) and the above equation we obtain that

$$
\begin{aligned}
\bar{I}_{r k}^{+}(h, \delta)= & -\frac{a_{0}^{r+1}(2 h)^{k+1 / 2}}{a_{1}^{(r+1) / 2}(2 k+2+r)} \\
& +\frac{(2 k+1)\left(2 h+a_{0}^{2} / a_{1}\right)}{2 k+2+r} \bar{I}_{r, k-1}^{+}(h, \delta), \quad k \geq 1, r \geq 0 .
\end{aligned}
$$

It follows that

$$
\begin{aligned}
\bar{I}_{r k}^{+}(h, \delta)= & -\frac{a_{0}^{r+1}}{a_{1}^{(r+1) / 2}} \sqrt{2 h} \widetilde{\varphi}_{r k}^{+}(h) \\
& +\widetilde{\alpha}_{r k}^{+}\left(2 h+\frac{a_{0}^{2}}{a_{1}}\right)^{k} \bar{I}_{r 0}^{+}(h, \delta), \quad k \geq 1, r \geq 0,
\end{aligned}
$$


where

$$
\begin{aligned}
\tilde{\alpha}_{r k}^{+}= & \frac{(2 k+1)(2 k-1)(2 k-3) \times \cdots \times 3}{(2 k+2+r)(2 k+r)(2 k-2+r) \times \cdots \times(4+r)}, \\
\widetilde{\varphi}_{r k}^{+}(h)= & \frac{(2 h)^{k}}{2 k+2+r}+\frac{2 k+1}{(2 k+2+r)(2 k+r)} \\
& \times\left(2 h+\frac{a_{0}^{2}}{a_{1}}\right)(2 h)^{k-1} \\
& +\frac{(2 k+1)(2 k-1)}{(2 k+2+r)(2 k+r)(2 k-2+r)} \\
& \times\left(2 h+\frac{a_{0}^{2}}{a_{1}}\right)^{2}(2 h)^{k-2}+\cdots \\
& +\frac{(2 k+1)(2 k-1) \times \cdots \times 5}{(2 k+2+r)(2 k+r)(2 k-2+r) \times \cdots \times(4+r)} \\
& \times\left(2 h+\frac{a_{0}^{2}}{a_{1}}\right)^{k-1} 2 h,
\end{aligned}
$$

which is a polynomial of degree $k$ in $h$. For convenience, introduce

$$
\bar{\varphi}_{r k}^{+}(h)=\left\{\begin{array}{ll}
\widetilde{\varphi}_{r k}^{+}(h), & k \geq 1, \\
0, & k=0,
\end{array} \quad \bar{\alpha}_{r k}^{+}= \begin{cases}\widetilde{\alpha}_{r k}^{+}, & k \geq 1, \\
1, & k=0 .\end{cases}\right.
$$

Then, combining (49) and (51) gives that

$$
\begin{aligned}
\bar{I}_{r k}^{+}(h, \delta)= & -\frac{a_{0}^{r+1}}{a_{1}^{(r+1) / 2}} \sqrt{2 h} \bar{\varphi}_{r k}^{+}(h) \\
& +\bar{\alpha}_{r k}^{+}\left(2 h+\frac{a_{0}^{2}}{a_{1}}\right)^{k} \bar{I}_{r 0}^{+}(h, \delta), \quad k \geq 0, r \geq 0 .
\end{aligned}
$$

Further, by using the formula

$$
\begin{aligned}
\int v^{r}\left(2 h+\frac{a_{0}^{2}}{a_{1}}-v^{2}\right)^{1 / 2} d v \\
=\frac{-v^{r-1}\left(2 h+a_{0}^{2} / a_{1}-v^{2}\right)^{3 / 2}}{r+2} \\
+\frac{(r-1)\left(2 h+a_{0}^{2} / a_{1}\right)}{r+2} \\
\quad \times \int v^{r-2}\left(2 h+\frac{a_{0}^{2}}{a_{1}}-v^{2}\right)^{1 / 2} d v,
\end{aligned}
$$

we have that

$$
\begin{aligned}
\bar{I}_{r 0}^{+}(h, \delta)= & \frac{a_{0}^{r-1}(2 h)^{3 / 2}}{a_{1}^{(r-1) / 2}(r+2)} \\
& +\frac{r-1}{r+2}\left(2 h+\frac{a_{0}^{2}}{a_{1}}\right) \bar{I}_{r-2,0}^{+}(h, \delta), \quad r \geq 1 .
\end{aligned}
$$

It follows that

$$
\begin{aligned}
\bar{I}_{r 0}^{+}(h, \delta)= & (2 h)^{3 / 2} \widetilde{\varphi}_{r}^{+}(h) \\
& +\widetilde{\alpha}_{r}^{+}\left(2 h+\frac{a_{0}^{2}}{a_{1}}\right)^{r / 2} \bar{I}_{00}(h, \delta), \quad r \geq 1,
\end{aligned}
$$

where

$$
\begin{aligned}
& \tilde{\alpha}_{r}^{+}= \begin{cases}0, & r \text { odd, } \\
\frac{(r-1)(r-3) \times \cdots \times 3 \times 1}{(r+2) r(r-2) \times \cdots \times 6 \times 4}, & r \text { even, }\end{cases} \\
& \widetilde{\varphi}_{r}^{+}(h)=\frac{a_{0}^{r-1}}{(r+2) a_{1}^{(r-1) / 2}}+\frac{(r-1) a_{0}^{r-3}}{(r+2) r a_{1}^{(r-3) / 2}}\left(2 h+\frac{a_{0}^{2}}{a_{1}}\right) \\
& +\frac{(r-1)(r-3) a_{0}^{r-5}}{(r+2) r(r-2) a_{1}^{(r-5) / 2}}\left(2 h+\frac{a_{0}^{2}}{a_{1}}\right)^{2}+\cdots \\
& +\left((r-1)(r-3) \times \cdots \times\left(r+1-2\left[\frac{(r-1)}{2}\right]\right)\right. \\
& \left.\times a_{0}^{r-1-2[(r-1) / 2]}\right) \\
& \times\left((r+2) r(r-2) \times \cdots \times\left(r+2-2\left[\frac{(r-2)}{2}\right]\right)\right. \\
& \left.\times a_{1}^{(r-1) / 2-[(r-1) / 2]}\left[\frac{(r-1)}{2}\right]\right)^{-1} \\
& \times\left(2 h+\frac{a_{0}^{2}}{a_{1}}\right)^{[(r-1) / 2]},
\end{aligned}
$$

which is a polynomial of degree $[(r-1) / 2]$ in $h$. Let

$$
\bar{\varphi}_{r}^{+}(h)=\left\{\begin{array}{ll}
\widetilde{\varphi}_{r}^{+}(h), & r \geq 1, \\
0, & r=0,
\end{array} \quad \bar{\alpha}_{r}^{+}= \begin{cases}\widetilde{\alpha}_{r}^{+}, & r \geq 1, \\
1, & r=0 .\end{cases}\right.
$$

Then, we have that by (55) and the above

$$
\begin{aligned}
\bar{I}_{r 0}^{+}(h, \delta)= & (2 h)^{3 / 2} \bar{\varphi}_{r}^{+}(h) \\
& +\bar{\alpha}_{r}^{+}\left(2 h+\frac{a_{0}^{2}}{a_{1}}\right)^{r / 2} \bar{I}_{00}^{+}(h, \delta), \quad r \geq 0 .
\end{aligned}
$$

Substituting the above equation into (52), one can find that

$$
\begin{aligned}
\bar{I}_{r k}^{+}(h, \delta)= & \sqrt{h} \bar{\psi}_{r k}^{+}(h)+\bar{\alpha}_{r k} \bar{\alpha}_{r}\left(2 h+\frac{a_{0}^{2}}{a_{1}}\right)^{k+r / 2} \\
& \times \bar{I}_{00}^{+}(h, \delta), \quad r \geq 0, \quad k \geq 0,
\end{aligned}
$$

where $\bar{\alpha}_{r}=0$ for $r$ odd, $\bar{\alpha}_{r}>0$ for $r$ even, and

$$
\begin{aligned}
\bar{\psi}_{r k}^{+}(h, \delta)= & -\frac{\sqrt{2} a_{0}^{r+1}}{a_{1}^{(r+1) / 2}} \bar{\varphi}_{r k}^{+}(h) \\
& +2 \sqrt{2} h \bar{\alpha}_{r k}\left(2 h+\frac{a_{0}^{2}}{a_{1}}\right)^{k} \bar{\varphi}_{r}^{+}(h),
\end{aligned}
$$


which is a polynomial of degree $k+[(r+1) / 2]$ in $h$. Combining (37), (45), and (59) gives that

$$
\begin{aligned}
M_{1}^{+}(h, \delta)= & \sqrt{h} \sum_{i+2 k=0}^{n-1} \frac{2 \bar{q}_{i, 2 k}^{+}}{\left(\sqrt{a_{1}}\right)^{i+1}} \sum_{r=0}^{i} C_{i}^{r}\left(-\frac{a_{0}}{\sqrt{a_{1}}}\right)^{i-r} \bar{\psi}_{r k}^{+}(h) \\
& +\sum_{i+2 k=0}^{n-1} \frac{2 \bar{q}_{i, 2 k}^{+}}{\left(\sqrt{a_{1}}\right)^{i+1}} \\
& \times \sum_{r=0, r \text { even }}^{i} C_{i}^{r} \bar{\alpha}_{r k} \bar{\alpha}_{r}\left(-\frac{a_{0}}{\sqrt{a_{1}}}\right)^{i-r} \\
& \times\left(2 h+\frac{a_{0}^{2}}{a_{1}}\right)^{k+r / 2} \bar{I}_{00}^{+}(h, \delta),
\end{aligned}
$$

which implies (35), together with (13) and (15).

Note that

$$
\int \sqrt{a^{2}-x^{2}} d x=\frac{1}{2}\left[x \sqrt{a^{2}-x^{2}}+a^{2} \arcsin \frac{x}{|a|}\right] .
$$

Then, we have

$$
\begin{aligned}
\bar{I}_{00}^{+}(h, \delta)= & -\frac{a_{0}}{2 \sqrt{a_{1}}} \sqrt{2 h}+\frac{1}{2}\left(2 h+\frac{a_{0}^{2}}{a_{1}}\right) \\
& \times\left(\frac{\pi}{2}-\arcsin \frac{a_{0}}{\sqrt{2 a_{1} h+a_{0}^{2}}}\right) .
\end{aligned}
$$

Inserting the above formula into (35), we can obtain (36). Hence, the proof is finished.

Similar to Lemma 4, we can obtain the following lemma about $M^{-}(h, \delta)$.

Lemma 5. Let system (5b) satisfy (3) and (4). Then

(i) If $b_{1}>0, b_{0}=0, M^{\top}(h, \delta)$ in (11) has the expression

$$
M^{-}(h, \delta)=\sqrt{h} \sum_{i+2 k=0}^{n} A_{i, 2 k}^{-}(\sqrt{h})^{i+2 k},
$$

where

$$
\begin{aligned}
A_{0,2 k}^{-}= & -\frac{2(\sqrt{2})^{2 k+1} a_{0,2 k}^{-}}{2 k+1}, \\
A_{i, 2 k}^{-}= & \frac{2(\sqrt{2})^{2 k+1+i}}{\left(\sqrt{b_{1}}\right)^{i}}\left(b_{i-1,2 k+1}^{-}+\frac{i}{2 k+1} a_{i, 2 k}^{-}\right) \\
& \times \int_{-\pi / 2}^{0} \sin ^{i-1} \theta \cos ^{2 k+2} \theta d \theta, \quad 1 \leq i \leq n .
\end{aligned}
$$

(ii) If $b_{1}>0, b_{0} \neq 0, M^{-}(h, \delta)$ in (11) has the form

$$
\begin{aligned}
M^{-}(h, \delta)= & \sqrt{h}\left[\sum_{i+2 k=0}^{n-1}\left(b_{i, 2 k+1}^{-}+\frac{i+1}{2 k+1} a_{i+1,2 k}^{-}\right) \phi_{i k}^{-}(h)\right. \\
& \left.-\sum_{2 k=0}^{n} \frac{2^{k+1+1 / 2} a_{0,2 k}^{-}}{2 k+1} h^{k}\right] \\
& +\sum_{i+2 k=0}^{n-1}\left(b_{i, 2 k+1}^{-}+\frac{i+1}{2 k+1} a_{i+1,2 k}^{-}\right) \\
& \times \sum_{r=0, \text { reven }}^{i} \alpha_{i r k}^{-}\left(2 h+\frac{b_{0}^{2}}{b_{1}}\right)^{k+r / 2} \bar{I}_{00}^{-}(h, \delta),
\end{aligned}
$$

or

$$
\begin{aligned}
M^{-}(h, \delta)= & \sqrt{h} \psi_{[n / 2]}^{-}(h, \delta)+\left(2 h+\frac{b_{0}^{2}}{b_{1}}\right) \varphi_{[(n-1) / 2]}^{-} \\
& \times\left(2 h+\frac{b_{0}^{2}}{b_{1}}, \delta\right)\left(\frac{\pi}{2}+\arcsin \frac{b_{0}}{\sqrt{2 b_{1} h+b_{0}^{2}}}\right),
\end{aligned}
$$

where

$$
\bar{I}_{00}^{-}(h, \delta)=\int_{-\sqrt{2 h+b_{0}^{2} / b_{1}}}^{b_{0} / \sqrt{b_{1}}} \sqrt{2 h+\frac{b_{0}^{2}}{b_{1}}-v^{2}} d v,
$$

each $\alpha_{i r k}^{-}$is nonzero constant and $\phi_{i k}^{-}, \psi_{[n / 2]}^{-}, \varphi_{[(n-1) / 2]}^{-}$are polynomials of degree $k+[(i+1) / 2],[n / 2],[(n-1) / 2]$, respectively.

\section{Proof of Theorem 1}

In this section, we will prove the main results. Obviously, under (9) there are the following 9 subcases:

(1) $a_{1}=b_{1}=0, a_{0}>0, b_{0}<0$,

(2) $a_{1}>0, b_{1}>0, a_{0}=b_{0}=0$,

(3) $a_{1}>0, b_{0}<0, a_{0}=b_{1}=0$,

(4) $a_{1}>0, a_{0}>0, b_{1}=0, b_{0}<0$,

(5) $a_{1}>0, a_{0}>0, b_{1}>0, b_{0}=0$,

(6) $a_{1}>0, a_{0}>0, b_{1}>0, b_{0}<0$,

(7) $a_{0}>0, b_{1}>0, a_{1}=b_{0}=0$,

(8) $a_{1}=0, a_{0}>0, b_{1}>0, b_{0}<0$,

(9) $a_{1}>0, a_{0}=0, b_{1}>0, b_{0}<0$.

We only give the proof of Subcases 1, 2, 3, 4, 5, and 6. And the Subcases 7, 8, and 9 can be verified, similar to Subcases 3, 4 , and 5 , respectively. 
Subcase 1. $a_{1}=b_{1}=0, a_{0}>0, \quad b_{0}<0$. From (12) and Lemma 3, one can obtain that

$$
\begin{aligned}
M(h, \delta) & =M^{+}(h, \delta)+M^{-}(h, \delta) \\
& =h^{1 / 2} \sum_{i+2 k=0}^{n} B_{i, 2 k}^{+} h^{i+k}+h^{1 / 2} \sum_{i+2 k=0}^{n} B_{i, 2 k}^{-} h^{i+k} \\
& =h^{1 / 2} \sum_{i+2 k=0}^{n}\left(B_{i, 2 k}^{+}+B_{i, 2 k}^{-}\right) h^{i+k},
\end{aligned}
$$

which implies that $M(h, \delta)$ has at most $n$ isolated positive zeros for $h>0$. To show that this bound can be reached, take $a_{i j}^{-}=b_{i j}^{ \pm}=0, a_{i j}^{+}=0, j \geq 1$. Then, by (27) and (29), (69) has the form

$$
M(h, \delta)=h^{1 / 2} \sum_{i=0}^{n} B_{i 0}^{+} h^{i}
$$

where

$$
B_{00}^{+}=2^{1+1 / 2} a_{00}^{+}, \quad B_{i 0}^{+}=\frac{2^{1+1 / 2}}{a_{0}^{i}} \frac{(2 i) ! !}{(2 i+1) ! !} a_{i 0}^{+}, \quad i \geq 1 .
$$

Hence, using (70) we can take $a_{i 0}^{+}, i=0,1, \ldots, n$ as free parameters to produce $n$ simple positive zeros of $M(h, \delta)$ near $h=0$, which gives $n$ limit cycles correspondingly near the origin. Thus, $N(n) \geq n$ in this case. This ends the proof.

Subcase 2. $a_{1}>0, b_{1}>0, a_{0}=b_{0}=0$ Similar to the above and using (32) and (64), $M(h, \delta)$ in (12) has the expression of the form

$$
\begin{aligned}
M(h, \delta) & =\sum_{i+2 k=0}^{n} A_{i, 2 k}^{+}(\sqrt{h})^{2 k+1+i}+\sum_{i+2 k=0}^{n} A_{i, 2 k}^{-}(\sqrt{h})^{2 k+1+i} \\
& =\sqrt{h} \sum_{l=0}^{n} A_{l}(\sqrt{h})^{l},
\end{aligned}
$$

where $A_{l}=\sum_{i+2 k=l}\left(A_{i, 2 k}^{+}+A_{i, 2 k}^{-}\right)$. Further, taking $a_{i j}^{-}=b_{i j}^{ \pm}=$ $0, a_{i j}^{+}=0, j \geq 1$, then, by (34) and (65), $M(h, \delta)$ in (72) becomes

$$
M(h, \delta)=h^{1 / 2} \sum_{i=0}^{n} A_{i 0}^{+}(\sqrt{h})^{i}
$$

where

$$
\begin{aligned}
& A_{00}^{+}=\frac{2 a_{00}^{+}}{2 k+1} \\
& A_{i 0}^{+}=\frac{2 i a_{i 0}^{+}}{\left(\sqrt{a_{1}}\right)^{i}} \int_{0}^{\pi / 2} \sin ^{i-1} \theta \cos ^{2} \theta \mathrm{d} \theta, \quad i \geq 1 .
\end{aligned}
$$

Thus, from (72) and (73), we can discuss similar to Subcase 1. This finishes the proof.
Subcase 3. $a_{1}>0, b_{0}<0, a_{0}=b_{1}=0$ By Lemmas 3 , and 4 and (12), we can have that

$$
\begin{aligned}
M(h, \delta) & =\sum_{i+2 k=0}^{n} A_{i, 2 k}^{+}(\sqrt{h})^{2 k+1+i}+h^{1 / 2} \sum_{i+2 k=0}^{n} B_{i, 2 k}^{-} h^{i+k} \\
& =h^{1 / 2}\left(\sum_{i+2 k=0}^{n} A_{i, 2 k}^{+}(\sqrt{h})^{i+2 k}+\sum_{i+2 k=0}^{n} B_{i, 2 k}^{-} h^{i+k}\right) \\
& =h^{1 / 2} M^{*}(h, \delta) .
\end{aligned}
$$

Let us prove that $M^{*}(h, \delta)$ has at most $n+[(n+1) / 2]$ zeros on the open interval $(0,+\infty)$. For the purpose, let $\sqrt{h}=\lambda$. Then, for $n=2 l, l \geq 1, M^{*}(h, \delta)$ in (75) has the expression

$$
\begin{aligned}
M^{*}(h, \delta) & =\sum_{i+2 k=0}^{n} A_{i, 2 k}^{+} \lambda^{i+2 k}+\sum_{i+2 k=0}^{n} B_{i, 2 k}^{-} \lambda^{2 i+2 k} \\
& =\sum_{j=0}^{2 l} C_{j} \lambda^{j}+\sum_{j=1}^{l} C_{2 l+j} \lambda^{2 j+2 l} \triangleq \bar{M}(\lambda, \delta),
\end{aligned}
$$

where

$$
\begin{gathered}
C_{j}=\sum_{i+2 k=j} A_{i, 2 k}^{+}+\sum_{2 i+2 k=j} B_{i, 2 k}^{-}, \quad j=0,1,2, \ldots, 2 l, \\
C_{2 l+j}=\sum_{i+k=j+l} B_{i, 2 k}^{-}, \quad j=1,2, \ldots, l .
\end{gathered}
$$

To prove $M^{*}(h, \delta)$ has at most $n+[(n+1) / 2]$ zeros, it suffices to prove $\bar{M}(\lambda, \delta)$ has at most $n+[(n+1) / 2]=3 l$ zeros for $\lambda>0$. By Rolles theorem we need only to prove that $d^{2 l} \bar{M}(\lambda, \delta) / d \lambda^{2 l}$ has at most $l$ zeros for $\lambda \in(0,+\infty)$. From (76), we can have that

$$
\frac{d^{2 l} \bar{M}(\lambda, \delta)}{d \lambda^{2 l}}=C_{2 l}(2 l) !+\sum_{j=1}^{l} C_{2 l+j} A_{2 l+2 j}^{2 l} \lambda^{2 j}
$$

which shows that $d^{2 l} \bar{M}(\lambda, \delta) / d \lambda^{2 l}$ has at most $l$ zeros for $\lambda>$ 0 . Thus, $M(h, \delta)$ has at most $3 l$ zeros for $h>0$. To prove $3 l$ zeros can appear, we only need to prove that $M^{*}(h, \delta)$ in (75) can appear $3 l$ zeros for $h>0$ small. Let $b_{i j}^{ \pm}=0, a_{i j}^{ \pm}=0, j \geq$ $1, a_{i 0}^{-}=0,0 \leq i \leq l$, and $a_{2 l}^{-} \neq 0$. Then $M^{*}(h, \delta)$ in (75) can be expressed as by (29) and (34)

$$
\begin{aligned}
M^{*}(h, \delta)= & \sum_{i=0}^{2 l} A_{i 0}^{+}(\sqrt{h})^{i}+\sum_{i=l+1}^{2 l} B_{i 0}^{-}(\sqrt{h})^{2 i} \\
= & A_{00}^{+}+A_{10}^{+} \sqrt{h}+A_{20}^{+}(\sqrt{h})^{2}+\cdots \\
& +A_{2 l-1,0}^{+}(\sqrt{h})^{2 l-1}+A_{2 l, 0}^{+}(\sqrt{h})^{2 l} \\
& +B_{l+1,0}^{-}(\sqrt{h})^{2 l+2}+B_{l+2,0}^{-}(\sqrt{h})^{2 l+4} \\
& +\cdots+B_{2 l, 0}^{-}(\sqrt{h})^{4 l},
\end{aligned}
$$


where

$$
\begin{aligned}
A_{00}^{+} & =2 \sqrt{2} a_{00}^{+}, \\
A_{i 0}^{+} & =\frac{2(\sqrt{2})^{i+1}}{\left(\sqrt{a_{1}}\right)^{i}} i a_{i 0}^{+}, \quad i=1,2, \ldots, 2 l, \\
B_{i 0}^{-} & =\frac{-2 \sqrt{2}}{b_{0}^{i}} a_{i 0}^{-} \int_{0}^{\pi / 2} \cos ^{2 i+1} \theta d \theta \\
& =\frac{-2 \sqrt{2}}{b_{0}^{i}} \frac{(2 i) ! !}{(2 i+1) ! !} a_{i 0}^{-}, \quad i=l+1, \ldots, 2 l .
\end{aligned}
$$

Thus, by changing the sign of $a_{2 l, 0}^{-}, a_{2 l-1,0}^{-}, \ldots, a_{l+1,0}^{-}, a_{2 l, 0}^{+}$, $a_{2 l-1,0}^{+}, \ldots, a_{00}^{+}$in turn such that

$$
\begin{gathered}
a_{i-1,0}^{-} a_{i 0}^{-}<0, \quad i=2 l, 2 l-1, \ldots, l+2, \\
a_{l+1}^{-} a_{2 l, 0}^{+}>0, \quad a_{i-1,0}^{+} a_{i 0}^{+}<0, \quad i=2 l, 2 l-1, \ldots, 1, \\
0<\left|a_{00}^{+}\right| \ll\left|a_{10}^{+}\right| \ll \cdots \ll\left|a_{2 l, 0}^{+}\right| \\
\ll\left|a_{l+1}^{-}\right| \ll\left|a_{l+2}^{-}\right| \ll\left|a_{2 l-1}^{-}\right| \ll 1,
\end{gathered}
$$

we can find $3 l$ simply positive zeros $h_{1}, h_{2}, \ldots, h_{3 l}$ with $0<$ $h_{3 l}<h_{3 l-1}<\cdots<h_{1} \ll 1$. For $n=2 l+1, l=0,1, \ldots$, we can discuss in a similar way. Thus, this bound can be reached and $N(n) \geq n+[(n+1) / 2]$. The proof is finished.

Subcase 4. $a_{1}>0, a_{0}>0, b_{1}=0, b_{0}<0$ From (12) and Lemmas 3 and 4 , we get that

$$
\begin{aligned}
M(h, \delta)= & \sqrt{h} \psi_{[n / 2]}^{+}(h, \delta)+\left(2 h+\frac{a_{0}^{2}}{a_{1}}\right) \varphi_{[(n-1) / 2]}^{+} \\
& \times\left(2 h+\frac{a_{0}^{2}}{a_{1}}, \delta\right)\left(\pi-2 \arcsin \frac{a_{0}}{\sqrt{2 a_{1} h+a_{0}^{2}}}\right) \\
& +\sqrt{h} \sum_{i+2 k=0}^{n} B_{i, 2 k}^{-} h^{i+k} \\
= & \sqrt{h} f_{n}(h, \delta)+\left(2 h+\frac{a_{0}^{2}}{a_{1}}\right) \varphi_{[(n-1) / 2]}^{+} \\
& \times\left(2 h+\frac{a_{0}^{2}}{a_{1}}, \delta\right)\left(\pi-2 \arcsin \frac{a_{0}}{\sqrt{2 a_{1} h+a_{0}^{2}}}\right),
\end{aligned}
$$

where

$$
f_{n}(h, \delta)=\psi_{[n / 2]}^{+}(h, \delta)+\sum_{i+2 k=0}^{n} B_{i, 2 k}^{-} h^{i+k},
$$

which is a polynomial of degree $n$ in $h$. Let $\lambda=\sqrt{h}$. Then $h=\lambda^{2}, \lambda \in(0,+\infty)$, and (82) becomes

$$
\begin{aligned}
M(h, \delta)= & \lambda f_{n}\left(\lambda^{2}\right) \\
& +v(\lambda, \delta)\left(\frac{\pi}{2}-\arcsin \frac{a_{0}}{\sqrt{2 a_{1} \lambda^{2}+a_{0}^{2}}}\right) \\
\triangleq & \widetilde{M}(\lambda, \delta),
\end{aligned}
$$

where

$$
v(\lambda, \delta)=\left(2 \lambda^{2}+\frac{a_{0}^{2}}{a_{1}}\right) \varphi_{[(n-1) / 2]}^{+}\left(2 \lambda^{2}+\frac{a_{0}^{2}}{a_{1}}, \delta\right) .
$$

One can see that $(d / d \lambda)(\widetilde{M}(\lambda, \delta) / v(\lambda, \delta))=u(\lambda, \delta) / v^{2}(\lambda, \delta)$, where

$$
\begin{aligned}
u(\lambda, \delta)= & \left(2 \lambda^{2}+\frac{a_{0}^{2}}{a_{1}}\right) \varphi_{[(n-1) / 2]}^{+}\left(2 \lambda^{2}+\frac{a_{0}^{2}}{a_{1}}, \delta\right) \\
& \times \frac{d}{d \lambda}\left(\lambda f_{n}\left(\lambda^{2}, \delta\right)\right)-\lambda f_{n}\left(\lambda^{2}, \delta\right) \\
& \times \frac{d}{d \lambda}\left[\left(2 \lambda^{2}+\frac{a_{0}^{2}}{a_{1}}\right) \varphi_{[(n-1) / 2]}^{+}\left(2 \lambda^{2}+\frac{a_{0}^{2}}{a_{1}}, \delta\right)\right] \\
& +\frac{\sqrt{2} a_{0}}{\sqrt{a_{1}}}\left(2 \lambda^{2}+\frac{a_{0}^{2}}{a_{1}}\right) \\
& \times\left(\varphi_{[(n-1) / 2]}^{+}\left(2 \lambda^{2}+\frac{a_{0}^{2}}{a_{1}}, \delta\right)\right)^{2} .
\end{aligned}
$$

Denote by $\#\{\lambda \in(0,+\infty) \mid f(\lambda)=0\}$ the number of zeros of the function in the interval $(0,+\infty)$ taking into account their multiplicities. Note that

$$
\operatorname{deg} v=2\left[\frac{n+1}{2}\right], \quad \operatorname{deg} u=2\left(n+\left[\frac{n+1}{2}\right]\right),
$$

and they are even functions in $\lambda$. Therefore,

$$
\begin{gathered}
\#\{\lambda \in(0,+\infty) \mid v(\lambda, \delta)\} \leq\left[\frac{n-1}{2}\right], \\
\#\{\lambda \in(0,+\infty) \mid u(\lambda, \delta)\} \leq n+\left[\frac{n+1}{2}\right] .
\end{gathered}
$$

Then, from [8], we can obtain that

$$
\begin{aligned}
\#\{\lambda \in & (0,+\infty) \mid \widetilde{M}(\lambda, \delta)=0\} \\
\leq & \#\{\lambda \in(0,+\infty) \mid v(\lambda, \delta)\} \\
& +\#\{\lambda \in(0,+\infty) \mid u(\lambda, \delta)\}+1 \\
\leq & {\left[\frac{n-1}{2}\right]+n+\left[\frac{n+1}{2}\right]+1 } \\
= & n+2\left[\frac{n+1}{2}\right],
\end{aligned}
$$


which implies that $Z(n) \leq n+2[(n+1) / 2]$. Now, we verify $Z(n) \geq n+[(n+1) / 2]$.

Make the transformation $u=\sqrt{v^{2} / 2 h-a_{0}^{2} / 2 a_{1} h}$. Then $\bar{I}_{00}^{+}(h, \delta)$ in $(35)$ becomes

$$
\bar{I}_{00}^{+}(h, \delta)=\frac{(2 h)^{3 / 2}}{\sqrt{a_{0}^{2} / a_{1}}} \int_{0}^{1} \frac{u\left(1-u^{2}\right)^{1 / 2}}{\sqrt{1+\left(2 h a_{1} u^{2} / a_{0}^{2}\right)}} d u
$$

which follows that as $h>0$ small

$$
\begin{aligned}
\bar{I}_{00}^{+}(h, \delta) & \\
= & \frac{(2 h)^{3 / 2}}{\sqrt{a_{0}^{2} / a_{1}}} \int_{0}^{1} u\left(1-u^{2}\right)^{1 / 2} \\
& \times\left[1+\sum_{m=1}^{+\infty} \frac{(-1)^{m}(2 m-1) ! !}{(2 m) ! !}\left(\frac{2 a_{1}}{a_{0}^{2}}\right)^{m} h^{m} u^{2 m}\right] d u .
\end{aligned}
$$

Note that

$$
\begin{aligned}
\int_{0}^{1} u^{2 m+1}\left(1-u^{2}\right)^{1 / 2} d u \\
\quad=\int_{0}^{\pi / 2} \sin ^{2 m+1} \theta \cos ^{2} \theta d \theta \quad(\text { Let } u=\sin \theta) \\
=\frac{(2 m) ! !}{(2 m+3) ! !}, \quad m=0,1,2, \ldots
\end{aligned}
$$

Inserting the above formula into (91) gives that

$$
\bar{I}_{00}(h, \delta)=2 \sqrt{h} \sqrt{\frac{2 a_{1}}{a_{0}^{2}}} \sum_{m=0}^{+\infty} C_{m}\left(\frac{2 a_{1}}{a_{0}^{2}}\right)^{m} h^{m+1},
$$

where

$$
C_{m}=\frac{(-1)^{m}}{(2 m+1)(2 m+3)}, \quad m \geq 0
$$

Take $b_{i j}^{-}=a_{i j}^{+}=0, a_{i j}^{-}=0, j \geq 1, b_{i j}^{+}=0, i \geq 1$. Then, by (28), (35), and (93), we can obtain that for $h>0$ small

$$
\begin{aligned}
M(h, \delta)=\sqrt{h}[ & \sum_{i=0}^{n} \frac{-2 \sqrt{2}(2 i) ! !}{(2 i+1) ! ! b_{0}^{i}} a_{i 0}^{-} h^{i}+\sum_{2 k=0}^{n-1} b_{0,2 k+1}^{+} \psi_{0 k}^{+}(h) \\
& +\sum_{2 k=0}^{n-1} 2^{k+1} \alpha_{00 k}^{+} \sqrt{\frac{2 a_{1}}{a_{0}^{2}}} b_{0,2 k+1}^{+}\left(h+\frac{a_{0}^{2}}{2 a_{1}}\right)^{k} \\
& \left.\times \sum_{m=0}^{+\infty} C_{m}\left(\frac{2 a_{1}}{a_{0}^{2}}\right)^{m} h^{m+1}\right] \\
= & \sqrt{h} \sum_{i \geq 0} v_{i} h^{i}
\end{aligned}
$$

where

$$
\begin{aligned}
v_{i}= & \frac{-2 \sqrt{2}(2 i) ! !}{(2 i+1) ! ! b_{0}^{i}} a_{i 0}^{-} \\
& +L_{i}\left(b_{01}^{+}, b_{03}^{+}, \ldots, b_{0,2[(n-1) / 2]+1}^{+}\right), \quad i=0,1,2, \ldots, n \\
v_{n+1+i}= & \sum_{2 k=0}^{n-1} \alpha_{00 k}^{+} 2^{k+1}\left(\frac{2 a_{1}}{a_{0}^{2}}\right)^{n+i-k+1 / 2} \\
& \times \sum_{r=0}^{k} C_{k}^{r} C_{n+i-r} b_{0,2 k+1}^{+}, \quad i \geq 0,
\end{aligned}
$$

with $L_{i}, i=0,1, \ldots, n$ being linear combination, $L_{i}(0,0, \ldots, 0)=0$. One can find that

$$
\frac{\partial\left(v_{0}, v_{1}, \ldots, v_{n}, v_{n+1}, v_{n+2}, \ldots, v_{n+[(n+1) / 2]}\right)}{\partial\left(a_{00}^{-}, a_{10}^{-}, \ldots, a_{n 0}^{-}, b_{01}^{+}, b_{03}^{+}, \ldots, b_{0,2[(n-1) / 2]+1}^{+}\right)}
$$

$$
=\left(\begin{array}{cc}
A_{1} & A_{2} \\
0 & 2 \sqrt{\frac{2 a_{1}}{a_{0}^{2}}} A_{3}
\end{array}\right) \equiv A,
$$

where $A_{2}$ is a $(n+1) \times[(n+1) / 2]$ matrix,

$$
\begin{gathered}
A_{1}=\left(\begin{array}{ccccc}
-2 \sqrt{2} & 0 & 0 & \cdots & 0 \\
0 & \frac{-4 \sqrt{2}}{b_{0}} & 0 & \cdots & 0 \\
0 & 0 & \frac{-16 \sqrt{2}}{15 b_{0}^{2}} & \ldots & 0 \\
\vdots & \vdots & \vdots & \ddots & \vdots \\
0 & 0 & 0 & \cdots & \frac{-2 \sqrt{2}(2 n) ! !}{(2 n+1) ! ! b_{0}^{n}}
\end{array}\right), \\
A_{3}=\left(\alpha_{000}^{+} \beta_{0}, 2 \alpha_{001}^{+} \beta_{1}, 2^{2} \alpha_{002}^{+} \beta_{2}, \ldots,\right. \\
\left.2^{[(n-1) / 2]} \alpha_{00,[(n-1) / 2]}^{+} \beta_{[(n-1) / 2]}\right)
\end{gathered}
$$

with $\beta_{i}$ are $[(n+1) / 2] \times 1$ matrix satisfying

$$
\beta_{i}=\left(\begin{array}{c}
\left(\frac{2 a_{1}}{a_{0}^{2}}\right)^{n-i} \sum_{r=0}^{i} C_{i}^{r} C_{n-r} \\
\left(\frac{2 a_{1}}{a_{0}^{2}}\right)^{n+1-i} \sum_{r=0}^{i} C_{i}^{r} C_{n+1-r} \\
\vdots \\
\left(\frac{2 a_{1}}{a_{0}^{2}}\right)^{n+[(n-1) / 2]-i} \sum_{r=0}^{i} C_{i}^{r} C_{n+[(n-1) / 2]-r} \\
i=0,1,2, \ldots,\left[\frac{n-1}{2}\right] .
\end{array}\right.
$$


Hence, we can obtain that from (97)

$$
\begin{aligned}
|A| & =\left(2 \sqrt{\frac{2 a_{1}}{a_{0}^{2}}}\right)^{[(n+1) / 2]}\left|A_{1}\right|\left|A_{3}\right| \\
& =\left(2 \alpha_{0}^{+} \sqrt{\frac{2 a_{1}}{a_{0}^{2}}}\right)^{[(n+1) / 2][(n-1) / 2]} \prod_{i=0}^{i} 2^{+} \alpha_{00 i}^{+} \prod_{i=0}^{n} \frac{-2 \sqrt{2}(2 i) ! !}{(2 i+1) ! ! b_{0}^{i}}|B|,
\end{aligned}
$$

where

$$
B=\left(\beta_{0}, \beta_{1}, \ldots, \beta_{[(n-1) / 2]}\right),
$$

and $\beta_{i}$ are given in (99). We claim that $|A| \neq 0$. We only need to prove $|B| \neq 0$ by the above formula. Using elementary transformations to $|B|$ by multiplying $i$ th column by $\left(2 a_{1} / a_{0}^{2}\right)^{i-1}, i=2,3, \ldots,[(n+1) / 2]$, we can obtain that by (99) and (101)

$$
|B|=\left(\frac{2 a_{1}}{a_{0}^{2}}\right)^{n[(n+1) / 2]}\left|B_{1}\right|,
$$

where

$$
\left|B_{1}\right|=\left|\begin{array}{cccc}
C_{n} & \sum_{r=0}^{1} C_{1}^{r} C_{n-r} & \cdots & \sum_{r=0}^{[(n-1) / 2]} C_{[(n-1) / 2]}^{r} C_{n-r} \\
C_{n+1} & \sum_{r=0}^{1} C_{1}^{r} C_{n+1-r} & \cdots & \sum_{r=0}^{[(n-1) / 2]} C_{[(n-1) / 2]}^{r} C_{n+1-r} \\
\vdots & \vdots & \ddots & \vdots \\
C_{n+[(n-1) / 2]} & \sum_{r=0}^{1} C_{1}^{r} C_{n+[(n-1) / 2]-r} & \cdots & \sum_{r=0}^{[(n-1) / 2]} C_{[(n-1) / 2]}^{r} C_{n+[(n-1) / 2]-r}
\end{array}\right| .
$$

Now we will use elementary transformations to $B_{1}$ as follows.

(1) Add the first column multiplying by -1 to $i$ th column, $i=2,3, \ldots,[(n+1) / 2]$.

(2) Add the second column multiplying by $-C_{i-1}^{1}$ to $i$ th column, $i=3,4, \ldots,[(n+1) / 2]$.
(3) Add the third column multiplying by $-C_{i-1}^{2}$ to $i$ th column, $i=4,5, \ldots,[(n+1) / 2]$

$[(n-1) / 2]$. Add the $[(n-1) / 2]$ th column multiplying by $-C_{[(n-1) / 2]}^{[(n-3) / 2]}$ to $[(n+1) / 2]$ th column,

$[(n+1) / 2]$. multiply $i$ th column by $(-1)^{i-1}, i=$ $2,3, \ldots,[(n+1) / 2]$.

Then, $\left|B_{1}\right|$ becomes, together with (94)

$$
\begin{aligned}
\left|B_{1}\right| & =(-1)^{[(n-1) / 2][(n+1) / 2] / 2} \operatorname{det}\left(\widetilde{\beta}_{0}, \widetilde{\beta}_{1}, \ldots, \widetilde{\beta}_{[(n-1) / 2]}\right) \\
& =2^{[(n+1) / 2]}(-1)^{n[(n+1) / 2]} \operatorname{det}\left(\bar{\beta}_{0}, \bar{\beta}_{1}, \ldots, \bar{\beta}_{[(n-1) / 2]}\right) \\
& \triangleq 2^{[(n+1) / 2]}(-1)^{n[(n+1) / 2]}\left|B_{2}\right|,
\end{aligned}
$$

where

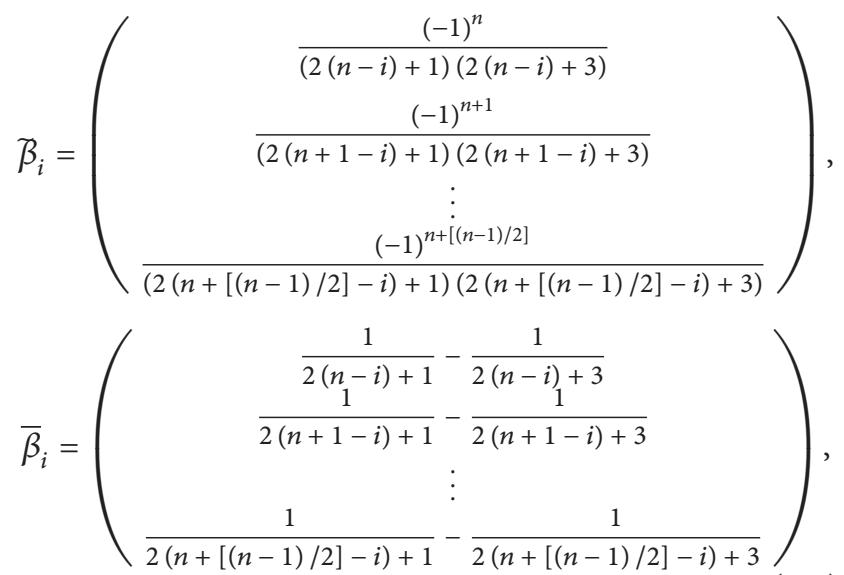

with $i=0,1, \ldots,[(n-1) / 2]$. For $B_{2}$ in (104) by adding a column on the left and a row on the above, we can obtain that, together with adding $i$ th column to $(i+1)$ th column with $i=1,2, \ldots,[(n+1) / 2]$

$$
\left|B_{2}\right|=\left|\begin{array}{ccccc}
1 & 1 & 1 & \cdots & 1 \\
\frac{1}{2 n+3} & \frac{1}{2 n+1} & \frac{1}{2 n-1} & \cdots & \frac{1}{2(n-[(n-1) / 2])+1} \\
\frac{1}{2 n+5} & \frac{1}{2 n+3} & \frac{1}{2 n+1} & \cdots & \frac{1}{2(n-[(n-1) / 2])+3} \\
\vdots & \vdots & \vdots & \ddots & \vdots \\
\frac{1}{2(n+[(n-1) / 2])+3} & \frac{1}{2(n+[(n-1) / 2])+1} & \frac{1}{2(n+[(n-1) / 2])-1} & \cdots & \frac{1}{2 n+1}
\end{array}\right|,
$$


which implies that $\left|A_{3}\right| \neq 0$ by (102) and (104) if $\left|B_{2}\right| \neq 0$. We claim that $\left|B_{2}\right| \neq 0$ and

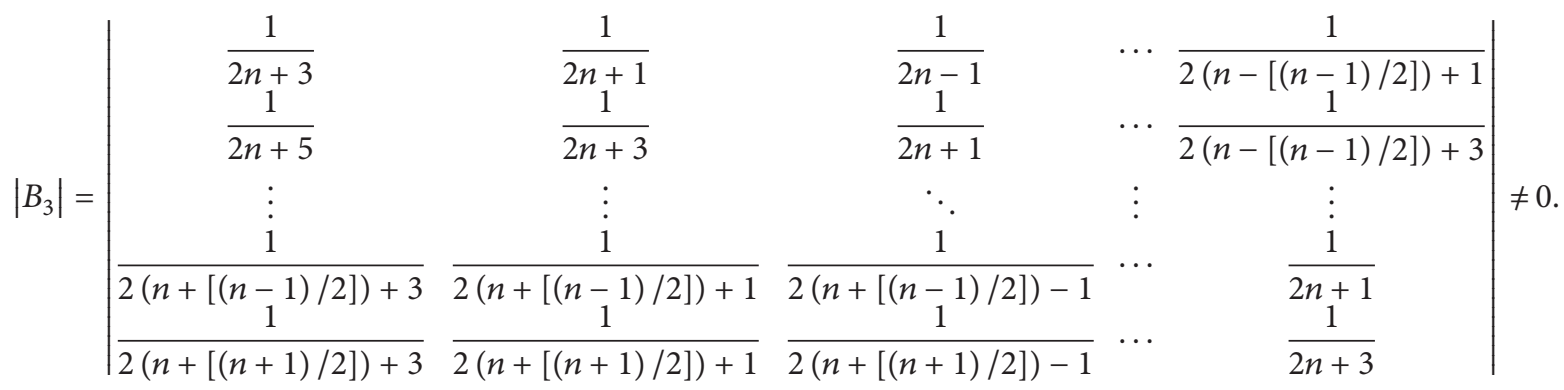

Now, we prove them by induction on $n$. For $n=1,2$ we have

$$
\begin{gathered}
\left|\begin{array}{ll}
1 & 1 \\
\frac{1}{5} & \frac{1}{3}
\end{array}\right|=\frac{2}{15}, \quad\left|\begin{array}{ll}
1 & 1 \\
\frac{1}{7} & \frac{1}{5}
\end{array}\right|=\frac{2}{35}, \\
\left|\begin{array}{ll}
\frac{1}{5} & \frac{1}{3} \\
\frac{1}{7} & \frac{1}{5}
\end{array}\right|=\frac{-4}{525}, \quad\left|\begin{array}{ll}
\frac{1}{7} & \frac{1}{5} \\
\frac{1}{9} & \frac{1}{7}
\end{array}\right|=\frac{-4}{2205},
\end{gathered}
$$

which means that (106) and (107) hold for $n=1,2$. Suppose (106) and (107) hold for $n=2 l-1,2 l, l \geq 1$. That is, we have for $n=2 l$

$$
\begin{gathered}
\left|B_{2}\right|=\left|\begin{array}{ccccc}
1 & 1 & 1 & \cdots & 1 \\
\frac{1}{4 l+3} & \frac{1}{4 l+1} & \frac{1}{4 l-1} & \cdots & \frac{1}{2 l+3} \\
\frac{1}{4 l+5} & \frac{1}{4 l+3} & \frac{1}{4 l+1} & \cdots & \frac{1}{2 l+5} \\
\vdots & \vdots & \vdots & \ddots & \vdots \\
\frac{1}{6 l+1} & \frac{1}{6 l-1} & \frac{1}{6 l-3} & \cdots & \frac{1}{4 l+1}
\end{array}\right| \triangleq|C| \neq 0, \\
\left|B_{3}\right|=\left|\begin{array}{cccccc}
\frac{1}{4 l+3} & \frac{1}{4 l+1} & \frac{1}{4 l-1} & \cdots & \frac{1}{2 l+3} \\
\frac{1}{4 l+5} & \frac{1}{4 l+3} & \frac{1}{4 l+1} & \cdots & \frac{1}{2 l+5} \\
\vdots & \vdots & \ddots & \vdots & \vdots \\
\frac{1}{6 l+1} & \frac{1}{6 l-1} & \frac{1}{6 l-3} & \cdots & \frac{1}{4 l+1} \\
\frac{1}{6 l+3} & \frac{1}{6 l+1} & \frac{1}{6 l-1} & \cdots & \frac{1}{4 l+3}
\end{array}\right| \neq 0 .
\end{gathered}
$$

Then for $n=2 l+1$, we have

$$
\begin{gathered}
\left|B_{2}\right|=\left|\begin{array}{ccccc}
1 & 1 & 1 & \cdots & 1 \\
\frac{1}{4 l+5} & \frac{1}{4 l+3} & \frac{1}{4 l+1} & \cdots & \frac{1}{2 l+3} \\
\frac{1}{4 l+7} & \frac{1}{4 l+5} & \frac{1}{4 l+3} & \cdots & \frac{1}{2 l+5} \\
\vdots & \vdots & \vdots & \ddots & \vdots \\
\frac{1}{6 l+5} & \frac{1}{6 l+3} & \frac{1}{6 l+1} & \cdots & \frac{1}{4 l+3}
\end{array}\right|, \\
\left|B_{3}\right|=\left|\begin{array}{ccccc}
\frac{1}{4 l+5} & \frac{1}{4 l+3} & \frac{1}{4 l+1} & \cdots & \frac{1}{2 l+3} \\
\frac{1}{4 l+7} & \frac{1}{4 l+5} & \frac{1}{4 l+3} & \cdots & \frac{1}{2 l+5} \\
\vdots & \vdots & \ddots & \vdots & \vdots \\
\frac{1}{6 l+5} & \frac{1}{6 l+3} & \frac{1}{6 l+1} & \cdots & \frac{1}{4 l+3} \\
\frac{1}{6 l+7} & \frac{1}{6 l+5} & \frac{1}{6 l+3} & \cdots & \frac{1}{4 l+5}
\end{array}\right| .
\end{gathered}
$$

Note that by the first equation of (109) there only exist $\alpha_{1}, \alpha_{2}, \ldots, \alpha_{1+1}$ such that

$$
\begin{aligned}
\left|B_{2}\right| & =\left[\frac{1}{6 l+5}-\left(\frac{\alpha_{1}}{6 l+3}+\frac{\alpha_{2}}{6 l+1}+\cdots+\frac{\alpha_{l+1}}{4 l+3}\right)\right]|C| \\
& =\frac{-2}{6 l+5}\left[\frac{\alpha_{1}}{6 l+3}+\frac{2 \alpha_{2}}{6 l+1}+\cdots+\frac{(l+1) \alpha_{l+1}}{4 l+3}\right]|C|
\end{aligned}
$$

since $\alpha_{1}+\alpha_{2}+\cdots+\alpha_{1+1}=1$, where $C$ is given in (109). If $\left|B_{2}\right|=0$, then we can obtain that from (109) and (111)

$$
\frac{\alpha_{1}}{6 l+3}+\frac{2 \alpha_{2}}{6 l+1}+\cdots+\frac{(l+1) \alpha_{l+1}}{4 l+3}=0 .
$$

Note that

$$
\begin{gathered}
\frac{\alpha_{1}}{4 l+3}+\frac{\alpha_{2}}{4 l+1}+\cdots+\frac{\alpha_{l+1}}{2 l+3}=\frac{1}{4 l+5}, \\
\frac{\alpha_{1}}{4 l+5}+\frac{\alpha_{2}}{4 l+3}+\cdots+\frac{\alpha_{l+1}}{2 l+5}=\frac{1}{4 l+7}, \\
\vdots \\
\frac{\alpha_{1}}{6 l+1}+\frac{\alpha_{2}}{6 l-1}+\cdots+\frac{\alpha_{l+1}}{4 l+1}=\frac{1}{6 l+3},
\end{gathered}
$$

which follows that, together with (112) 


$$
\begin{aligned}
& \frac{\alpha_{1}}{4 l+3}+\frac{2 \alpha_{2}}{4 l+1}+\cdots+\frac{(l+1) \alpha_{l+1}}{2 l+3}=0 \\
& \frac{\alpha_{1}}{6 l+1}+\frac{2 \alpha_{2}}{6 l-1}+\cdots+\frac{(l+1) \alpha_{l+1}}{4 l+1}=0
\end{aligned}
$$

By the second equation in (109) and the above formula, we have

$$
\alpha_{1}=\alpha_{2}=\cdots=\alpha_{1+1}=0
$$

This is a contradiction with $\alpha_{1}+\alpha_{2}+\cdots+\alpha_{l+1}=1$. Hence $\alpha_{1} /(6 l+3)+2 \alpha_{2} /(6 l+1)+\cdots+(l+1) \alpha_{l+1} /(4 l+3) \neq 0$, which means that (106) holds for $n=2 l+1$. Since $\left|B_{2}\right| \neq 0$ in (110), there only exist $\beta_{1}, \beta_{2}, \ldots, \beta_{l+1}$ such that

$$
\begin{gathered}
\beta_{1}+\frac{\beta_{2}}{4 l+5}+\frac{\beta_{3}}{4 l+7}+\cdots+\frac{\beta_{l+1}}{6 l+5}=\frac{1}{6 l+7}, \\
\beta_{1}+\frac{\beta_{2}}{4 l+3}+\frac{\beta_{3}}{4 l+5}+\cdots+\frac{\beta_{l+1}}{6 l+3}=\frac{1}{6 l+5}, \\
\vdots \\
\beta_{1}+\frac{\beta_{2}}{2 l+3}+\frac{\beta_{3}}{2 l+5}+\cdots+\frac{\beta_{l+1}}{4 l+3}=\frac{1}{4 l+5},
\end{gathered}
$$

with $\beta_{1} \neq 0$ since the last row in the second formula of (110) is linearly independent with all rows in the first formula of (110), which means that (107) holds for $n=2 l+1$. In a similar way, we can prove (106) and (107) hold for $n=2 l+2$. Hence, the claim holds. So, from (99) we can know that $a_{00}^{-}, a_{10}^{-}, \ldots, a_{n 0}^{-}, b_{01}^{+}, b_{03}^{+}, \ldots, b_{0,2[(n-1) / 2]+1}^{+}$can be taken as free parameters. So we can choose these values such that

$$
\begin{aligned}
v_{i} v_{i+1} & <0, \quad i=0,1, \ldots, n+\left[\frac{(n-1)}{2}\right], \\
0 & <\left|v_{0}\right| \ll\left|v_{1}\right| \ll \cdots \ll\left|v_{n+[(n+1) / 2]}\right| \ll 1,
\end{aligned}
$$

which yields that by $(97)$ and $(96) M(h, \delta)$ can appear $n+[(n+$ $1) / 2$ ] positive zeros for $h>0$ small. We also can know that $N(n) \geq n+[(n+1) / 2]$. Hence, the conclusion is proved.

Subcase 5. $a_{1}>0, a_{0}>0, b_{1}>0, b_{0}=0$ By (36) and (67), one can see that

$$
\begin{aligned}
M(h, \delta)= & \sqrt{h} \psi_{[n / 2]}^{+}(h, \delta)+\left(2 h+\frac{a_{0}^{2}}{a_{1}}\right) \varphi_{[(n-1) / 2]}^{+} \\
& \times\left(2 h+\frac{a_{0}^{2}}{a_{1}}, \delta\right)\left(\frac{\pi}{2}-\arcsin \frac{a_{0}}{\sqrt{2 a_{1} h+a_{0}^{2}}}\right)
\end{aligned}
$$

$$
\begin{aligned}
& \frac{\alpha_{1}}{4 l+5}+\frac{2 \alpha_{2}}{4 l+3}+\cdots+\frac{(l+1) \alpha_{l+1}}{2 l+5}=0 \\
& \frac{\alpha_{1}}{6 l+3}+\frac{2 \alpha_{2}}{6 l+1}+\cdots+\frac{(l+1) \alpha_{l+1}}{4 l+3}=0 .
\end{aligned}
$$

$$
\begin{aligned}
& +\sqrt{h} \sum_{i+2 k=0}^{n} A_{i, 2 k}^{-}(\sqrt{h})^{i+2 k} \\
= & \sqrt{h} g_{[n / 2]}(h, \delta)+\bar{g}_{[(n+1) / 2]}(h, \delta) \\
& +\left(2 h+\frac{a_{0}^{2}}{a_{1}}\right) \varphi_{[(n-1) / 2]}^{+}\left(2 h+\frac{a_{0}^{2}}{a_{1}}, \delta\right) \\
& \times\left(\frac{\pi}{2}-\arcsin \frac{a_{0}}{\sqrt{2 a_{1} h+a_{0}^{2}}}\right),
\end{aligned}
$$

where

$$
\begin{gathered}
g_{[n / 2]}(h, \delta)=\psi_{[n / 2]}^{+}(h, \delta)+\sum_{l=0}^{[n / 2]} \sum_{i+2 k=2 l} A_{i, 2 k}^{-} h^{1}, \\
\bar{g}_{[(n+1) / 2]}(h, \delta)=\sum_{l=0}^{[(n-1) / 2]} \sum_{i+2 k=2 l+1} A_{i, 2 k}^{-} h^{l+1}, \quad n \geq 1 .
\end{gathered}
$$

For convenience, we denote by $g_{n}$ any polynomial of degree $n$ although its coefficients may be different when it appears in different place. Then, we claim that for any $2 \leq k \leq[(n-1) / 2]$

$$
\begin{aligned}
\frac{d^{k} M(h, \delta)}{d h^{k}}= & \sum_{j=0}^{k} h^{1 / 2-k+j} g_{[n / 2]-j}+\frac{d^{k} \bar{g}_{[(n+1) / 2]}}{d h^{k}} \\
& +\sum_{j=0}^{k-2} g_{j} h^{-j-1 / 2}\left(2 h+\frac{a_{0}^{2}}{a_{1}}\right)^{-j-1} \\
& +\frac{d^{k}}{d h^{k}}\left[\left(2 h+\frac{a_{0}^{2}}{a_{1}}\right) \varphi_{[(n-1) / 2]}^{+}\left(2 h+\frac{a_{0}^{2}}{a_{1}}, \delta\right)\right] \\
& \times\left(\frac{\pi}{2}-\arcsin \frac{a_{0}}{\sqrt{2 a_{1} h+a_{0}^{2}}}\right) .
\end{aligned}
$$


Now, we verify this claim by induction on $k$. For $k=2$, by (118) we can obtain that

$$
\begin{aligned}
& \frac{d M(h, \delta)}{d h}=\frac{1}{2} h^{-1 / 2} g_{[n / 2]}(h, \delta) \\
& +h^{1 / 2} \frac{d g_{[n / 2]}(h, \delta)}{d h}+\frac{d \bar{g}_{[(n+1) / 2]}}{d h} \\
& +\frac{d}{d h}\left[\left(2 h+\frac{a_{0}^{2}}{a_{1}}\right) \varphi_{[(n-1) / 2]}^{+}\left(2 h+\frac{a_{0}^{2}}{a_{1}}, \delta\right)\right] \\
& \times\left(\frac{\pi}{2}-\arcsin \frac{a_{0}}{\sqrt{2 a_{1} h+a_{0}^{2}}}\right) \\
& +\left(2 h+\frac{a_{0}^{2}}{a_{1}}\right) \varphi_{[(n-1) / 2]}^{+} \\
& \times\left(2 h+\frac{a_{0}^{2}}{a_{1}}, \delta\right) \frac{a_{0}}{\sqrt{2 a_{1}} \sqrt{h}\left(2 h+a_{0}^{2} / a_{1}\right)} \\
& =h^{-1 / 2} g_{[n / 2]}+h^{1 / 2} g_{[n / 2]-1}+\frac{d \bar{g}_{[(n+1) / 2]}}{d h} \\
& +\frac{d}{d h}\left[\left(2 h+\frac{a_{0}^{2}}{a_{1}}\right) \varphi_{[(n-1) / 2]}^{+}\left(2 h+\frac{a_{0}^{2}}{a_{1}}, \delta\right)\right] \\
& \times\left(\frac{\pi}{2}-\arcsin \frac{\mathrm{a}_{0}}{\sqrt{2 \mathrm{a}_{1} h+\mathrm{a}_{0}^{2}}}\right),
\end{aligned}
$$

which follows that

$$
\begin{aligned}
\frac{d^{2} M(h, \delta)}{d h^{2}}= & h^{-3 / 2} g_{[n / 2]}+h^{-1 / 2} g_{[n / 2]-1} \\
& +h^{1 / 2} g_{[n / 2]-2}+\frac{d^{2} \bar{g}_{[(n+1) / 2]}}{d h^{2}}+\frac{g_{0}}{\sqrt{h}\left(2 h+a_{0}^{2} / a_{1}\right)} \\
& +\frac{d^{2}}{d h^{2}}\left[\left(2 h+\frac{a_{0}^{2}}{a_{1}}\right) \varphi_{[(n-1) / 2]}^{+}\left(2 h+\frac{a_{0}^{2}}{a_{1}}, \delta\right)\right] \\
& \times\left(\frac{\pi}{2}-\arcsin \frac{a_{0}}{\sqrt{2 a_{1} h+a_{0}^{2}}}\right) .
\end{aligned}
$$

Hence, (120) holds for $k=2$. Suppose (120) holds for $k, 2 \leq$ $k \leq[(n-1) / 2]-1$. Then for $k+1$, we have

$$
\begin{aligned}
& \frac{d^{k+1} M(h, \delta)}{d h^{k+1}} \\
& =\sum_{j=0}^{k}\left(\frac{1}{2}-k+j\right) h^{1 / 2-(k+1)+j} g_{[n / 2]-j}
\end{aligned}
$$

$$
\begin{aligned}
& +\sum_{j=0}^{k} h^{1 / 2-k+j} g_{[n / 2]-1-j}+\frac{d^{k+1} \bar{g}_{[(n+1) / 2]}}{d h^{k+1}} \\
& +\frac{d^{k+1}}{d h^{k+1}}\left[\left(2 h+\frac{a_{0}^{2}}{a_{1}}\right) \varphi_{[(n-1) / 2]}^{+}\left(2 h+\frac{a_{0}^{2}}{a_{1}}, \delta\right)\right] \\
& \times\left(\frac{\pi}{2}-\arcsin \frac{a_{0}}{\sqrt{2 a_{1} h+a_{0}^{2}}}\right) \\
& +\frac{d^{k}}{d h^{k}}\left[\left(2 h+\frac{a_{0}^{2}}{a_{1}}\right) \varphi_{[(n-1) / 2]}^{+}\left(2 h+\frac{a_{0}^{2}}{a_{1}}, \delta\right)\right] \\
& \times \frac{a_{0}}{\sqrt{2 a_{1}} \sqrt{h}\left(2 h+a_{0}^{2} / a_{1}\right)} \\
& +\sum_{j=0}^{k-2}\left(h\left(2 h+\frac{a_{0}^{2}}{a_{1}}\right) \frac{d}{d h} g_{j}\left(2 h+\frac{a_{0}^{2}}{a_{1}}\right)^{j+2}\right)^{-1}, \\
& \left.\times\left(h^{j+3 / 2}\left(j+\frac{1}{2}\right)\left(2 h+\frac{a_{0}^{2}}{a_{1}}\right)\right]\right) \\
& -g_{j}[2(j+1) h+(j+1
\end{aligned}
$$

which implies that $(120)$ holds for $k+1$. Thus, the claim is proved. Then, taking $k=[(n-1) / 2]$, we can obtain that by differentiating it

$$
\begin{aligned}
\frac{d^{[(n+1) / 2]} M(h, \delta)}{d h^{[(n+1) / 2]}=} & \sum_{j=0}^{[n / 2]} h^{1 / 2-[(n+1) / 2]+j} g_{[n / 2]-j} \\
& +\sum_{j=0}^{[(n+1) / 2]-2} g_{j} h^{-j-1 / 2}\left(2 h+\frac{a_{0}^{2}}{a_{1}}\right)^{-j-1} \\
& +\frac{d^{[(n+1) / 2]} \bar{g}_{[(n+1) / 2]}}{d h^{[(n+1) / 2]}}+\frac{d^{[(n+1) / 2]}}{d h^{[(n+1) / 2]}} \\
& \times\left[\left(2 h+\frac{a_{0}^{2}}{a_{1}}\right) \varphi_{[(n-1) / 2]}^{+}\left(2 h+\frac{a_{0}^{2}}{a_{1}}, \delta\right)\right] \\
& \times\left(\frac{\pi}{2}-\arcsin \frac{a_{0}}{\sqrt{2 a_{1} h+a_{0}^{2}}}\right)
\end{aligned}
$$

One can find that

$$
\begin{aligned}
\frac{d^{[(n+1) / 2]+1} M(h, \delta)}{d h^{[(n+1) / 2]+1}=} & \sum_{j=0}^{[n / 2]} h^{1 / 2-[(n+1) / 2]-1+j} g_{[n / 2]-j} \\
& +\sum_{j=0}^{[(n+1) / 2]-1} g_{j} h^{-j-1 / 2}\left(2 h+\frac{a_{0}^{2}}{a_{1}}\right)^{-j-1}
\end{aligned}
$$




$$
\begin{aligned}
= & h^{-[(n+1) / 2]-1 / 2} \\
& \times\left(2 h+\frac{a_{0}^{2}}{a_{1}}\right)^{-[(n+1) / 2]} F(h, \delta),
\end{aligned}
$$

where

$$
\begin{aligned}
F(h, \delta)= & \left(2 h+\frac{a_{0}^{2}}{a_{1}}\right)^{[(n+1) / 2]} \sum_{j=0}^{[n / 2]} h^{j} g_{[n / 2]-j} \\
& +\sum_{j=0}^{[(n+1) / 2]-1} g_{j} h^{[(n+1) / 2]-j} \\
& \times\left(2 h+\frac{a_{0}^{2}}{a_{1}}\right)^{[(n+1) / 2]-j-1},
\end{aligned}
$$

where $F$ is a polynomial of degree $[n / 2]+[(n+1) / 2]$. Since $M(0, \delta)=0$ from (118), it is easy to see that $M(h, \delta)$ has at most $[n / 2]+2[(n+1) / 2]=n+[(n+1) / 2]$ zeros for $h>$ 0 by Rolle theorem. As the above discussion, we only prove $Z(n) \geq n+[(n+1) / 2]$ as $h>0$ small, which implies $N(n) \geq$ $n+[(n+1) / 2]$. For the purpose, take $b_{i j}^{-}=a_{i j}^{+}=0, a_{i j}^{-}=0, j \geq$ $1, b_{i j}^{+}=0, i \geq 1$. Then using (49), (51), we can write $M(h, \delta)$ in (12) as

$$
\begin{aligned}
M(h, \delta)=\sqrt{h}[ & -2 \sqrt{2} a_{00}^{-}+\sum_{i=1}^{n} \frac{2(\sqrt{2})^{i+1} i}{\left(\sqrt{b_{1}}\right)^{i}} a_{i 0}^{-} \\
& \times \int_{-\pi / 2}^{0} \sin ^{i-1} \theta \cos ^{2} \theta d \theta(\sqrt{h})^{i} \\
& +\sum_{2 k=0}^{n-1} b_{0,2 k+1}^{+} \psi_{0 k}^{+}(h) \\
& +\sum_{2 k=0}^{n-1} 2^{k+1} \alpha_{00 k}^{+} \sqrt{\frac{2 a_{1}}{a_{0}^{2}} b_{0,2 k+1}^{+}} \\
& \left.\times\left(h+\frac{a_{0}^{2}}{2 a_{1}}\right)^{k} \sum_{m=0}^{+\infty} C_{m}\left(\frac{2 a_{1}}{a_{0}^{2}}\right)^{m} h^{m+1}\right] .
\end{aligned}
$$

For $n=2 l, l \geq 1,(127)$ can be written as

$$
M(h, \delta)=\sqrt{h}\left[\sum_{i=0}^{2 l} \bar{v}_{i}(\sqrt{h})^{i}+\sum_{i \geq 0} \bar{v}_{2 l+1+i} h^{l+1+i}\right]
$$

where

$$
\begin{aligned}
& \bar{v}_{0}=- \sqrt{2} a_{00}^{-} . \\
& \bar{v}_{2 i+1}= \frac{2(\sqrt{2})^{2 i+2}(2 i+1)}{\left(\sqrt{b_{1}}\right)^{2 i+1}} a_{2 i+1,0}^{-} \\
& \quad \int_{-\pi / 2}^{0} \sin ^{2 i} \theta \cos ^{2} \theta d \theta, \quad i=0,1, \ldots, l-1, \\
& \bar{v}_{2 i}=\frac{2(\sqrt{2})^{2 i+1} 2 i}{\left(\sqrt{b_{1}}\right)^{2 i}} a_{2 i, 0}^{-} \int_{-\pi / 2}^{0} \sin ^{2 i-1} \theta \cos ^{2} \theta d \theta \\
&+L_{2 i}\left(b_{01}^{+}, b_{03}^{+}, \ldots, b_{0,2[(n-1) / 2]+1}^{+}\right), \quad i=1,2, \ldots, l, \\
& \bar{v}_{2 l+1+i}=\sum_{k=0}^{l-1} 2^{k+1} \alpha_{00 k}^{+}\left(\frac{2 a_{1}}{a_{0}^{2}}\right)^{l-k+i+1 / 2} \\
& \quad \times b_{0,2 k+1}^{+} \sum_{r=0}^{k} C_{k}^{r} C_{l+i-r}, \quad i=0,1, \ldots,\left[\frac{n-1}{2}\right],
\end{aligned}
$$

which implies that $M(h, \delta)$ can appear $n+[(n+1) / 2]$ zeros in $h>0$ small by using the same method with the Subcase 4 . For $n=2 l+1, l=0,1, \ldots$, we can discuss by (127) in a similar way. Hence, the conclusion holds.

Subcase 6. $a_{1}>0, a_{0}>0, b_{1}>0, b_{0}<0$. We have as the above

$$
\begin{aligned}
M(h, \delta)= & \sqrt{h}\left(\psi_{[n / 2]}^{+}(h, \delta)+\psi_{[n / 2]}^{-}(h, \delta)\right) \\
& +\left(2 h+\frac{a_{0}^{2}}{a_{1}}\right) \varphi_{[(n-1) / 2]}^{+}(h, \delta) \\
& \times\left(\frac{\pi}{2}-\arcsin \frac{a_{0}}{\sqrt{2 a_{1} h+a_{0}^{2}}}\right) \\
& +\left(2 h+\frac{b_{0}^{2}}{b_{1}}\right) \varphi_{[(n-1) / 2]}^{-}(h, \delta) \\
& \times\left(\frac{\pi}{2}+\arcsin \frac{b_{0}}{\sqrt{2 b_{1} h+b_{0}^{2}}}\right) .
\end{aligned}
$$

Similar to the Subcase 5 , we can prove that for any $2 \leq k \leq$ $[(n-1) / 2]$

$$
\begin{aligned}
& \frac{d^{k} M(h, \delta)}{d h^{k}} \\
& =\sum_{j=0}^{k} h^{1 / 2-k+j} g_{[n / 2]-j}
\end{aligned}
$$




$$
\begin{aligned}
& +\sum_{j=0}^{k-2} g_{j} h^{-j-1 / 2}\left(2 h+\frac{a_{0}^{2}}{a_{1}}\right)^{-j-1} \\
& +\sum_{j=0}^{k-2} g_{j} h^{-j-1 / 2}\left(2 h+\frac{b_{0}^{2}}{b_{1}}\right)^{-j-1} \\
& +\frac{d^{k}}{d h^{k}}\left[\left(2 h+\frac{a_{0}^{2}}{a_{1}}\right) \varphi_{[(n-1) / 2]}^{+}\left(2 h+\frac{a_{0}^{2}}{a_{1}}, \delta\right)\right] \\
& \times\left(\frac{\pi}{2}-\arcsin \frac{a_{0}}{\sqrt{2 a_{1} h+a_{0}^{2}}}\right) \\
& \left.+\frac{d^{k}}{d h^{k}}\left[\left(2 h+\frac{b_{0}^{2}}{b_{1}}\right) \varphi_{\left[\frac{(n-1)}{2}\right.}^{+}\right]\left(2 h+\frac{b_{0}^{2}}{a_{1}}, \delta\right)\right] \\
& \times\left(\frac{\pi}{2}-\arcsin \frac{b_{0}}{\left.\sqrt{2 b_{1} h+b_{0}^{2}}\right)} .\right.
\end{aligned}
$$

Taking $k=[(n-1) / 2]$ and differentiating the above twice follow that

$$
\begin{aligned}
\frac{d^{[(n+1) / 2]+1} M(h, \delta)}{d h^{[(n+1) / 2]+1}=} & \sum_{j=0}^{[n / 2]} h^{1 / 2-[(n+1) / 2]-1+j} g_{[n / 2]-j} \\
& +\sum_{j=0}^{[(n+1) / 2]-1} g_{j} h^{-j-1 / 2}\left(2 h+\frac{a_{0}^{2}}{a_{1}}\right)^{-j-1} \\
& +\sum_{j=0}^{[(n+1) / 2]-1} g_{j} h^{-j-1 / 2}\left(2 h+\frac{b_{0}^{2}}{b_{1}}\right)^{-j-1}
\end{aligned}
$$

If $a_{0}^{2} / a_{1}=b_{0}^{2} / b_{1}$, then it is easy to see that (132) has the same form with (125). Hence, we can know that $M(h, \delta)$ has at most $[n / 2]+2[(n+1) / 2]$ zeros for $h \in(0,+\infty)$. If $a_{0}^{2} / a_{1} \neq b_{0}^{2} / b_{1}$, then (132) can be written as

$$
\begin{aligned}
\frac{d^{[(n+1) / 2]+1} M(h, \delta)}{d h^{[(n+1) / 2]+1}=} & h^{-[(n+1) / 2]-1 / 2}\left(2 h+\frac{a_{0}^{2}}{a_{1}}\right)^{-[(n+1) / 2]} \\
& \times\left(2 h+\frac{b_{0}^{2}}{b_{1}}\right)^{-[(n+1) / 2]} \bar{F}(h, \delta),
\end{aligned}
$$

where

$$
\begin{aligned}
\bar{F}(h, \delta)= & \left(2 h+\frac{a_{0}^{2}}{a_{1}}\right)^{[(n+1) / 2]}\left(2 h+\frac{b_{0}^{2}}{b_{1}}\right)^{[(n+1) / 2]} \\
& \times \sum_{j=0}^{[n / 2]} h^{j} g_{[n / 2]-j} \sum_{j=0} g_{j} h^{[(n+1) / 2]-j} \\
& \left.+\left(2 h+\frac{b_{0}^{2}}{b_{1}}\right)^{[(n+1) / 2][(n+1) / 2]-1}\right) \\
& \left.+\left(2 h+\frac{a_{0}^{2}}{a_{1}}\right)^{[(n+1) / 2]-j-1} \sum_{j=0}^{a_{1}^{2}}\right)^{[(n+1) / 2][(n+1) / 2]-1} g_{j} h^{[(n+1) / 2]-j} \\
& \times\left(2 h+\frac{b_{0}^{2}}{b_{1}}\right)^{[(n+1) / 2]-j-1},
\end{aligned}
$$

where $\bar{F}$ is a polynomial of degree $[n / 2]+2[(n+1) / 2]$ in $h$. By Rolle theorem, we can obtain that $M(h, \delta)$ has at most $[n / 2]+$ $3[(n+1) / 2]$ zeros for $h>0$ since $M(0, \delta)=0$. Now, we only need to prove $Z(n) \geq n$. Take $a_{i j}^{-}=b_{i j}^{-}=0, a_{i j}^{+}=b_{i j}^{+}=0, i \geq$ 1. Then, by Lemmas 4,5 , one can see that for $h>0$ small

$$
\begin{aligned}
M(h, \delta)=\sqrt{h}\left[\sum_{2 k=0}^{n} \frac{2^{k+1+1 / 2} a_{0,2 k}^{+}}{2 k+1} h^{k}+\sum_{2 k=0}^{n-1} b_{0,2 k+1}^{+} \psi_{0 k}^{+}(h)\right. \\
+\sum_{2 k=0}^{n-1} 2^{k+1} \alpha_{00 k}^{+} \sqrt{\frac{2 a_{1}}{a_{0}^{2}} b_{0,2 k+1}^{+}\left(h+\frac{a_{0}^{2}}{2 a_{1}}\right)^{k}} \\
\left.\quad \times \sum_{m=0}^{+\infty} C_{m}\left(\frac{2 a_{1}}{a_{0}^{2}}\right)^{m} h^{m+1}\right] .
\end{aligned}
$$

Similarly, we can discuss the above formula such that such that $M(h, \delta)$ can appear $n$ zeros for $h>0$ small. Hence, the conclusion is proved.

\section{Acknowledgment}

The project was supported by National Natural Science Foundation of China (11271261), a grant from Ministry of Education of China (20103127110001) and FP7-PEOPLE2012-IRSES-316338.

\section{References}

[1] A. F. Filippov, Differential Equations with Discontinuous Righthand Sides, vol. 18, Kluwer Academic, Dodrecht, The Netherlands, 1988.

[2] M. Kunze, Non-Smooth Dynamical Systems, Springer, Berlin, Germany, 2000. 
[3] M. di Bernardo, C. J. Budd, A. R. Champneys, and P. Kowalczyk, Piecewise-Smooth Dynamical Systems, vol. 163, Springer, London, UK, 2008.

[4] M. S. Branicky, "Stability of swithed and hybrid systems," in Proceedings of the 33rd IEEE Conference on Decision Control, pp. 3498-3503, Lake Buena Vista, Fla, USA, December 1994.

[5] R. A. DeCarlo, M. S. Branicky, S. Pettersson, and B. Lennartson, "Perspectives and results on the stability and stabilizability of hybrid systems," Proceedings of IEEE, vol. 88, pp. 1069-1081, 2000.

[6] X.-S. Yang and G. Chen, "Limit cycles and chaotic invariant sets in autonomous hybrid planar systems," Nonlinear Analysis. Hybrid Systems, vol. 2, no. 3, pp. 952-957, 2008.

[7] X. Liu and M. Han, "Bifurcation of limit cycles by perturbing piecewise Hamiltonian systems," International Journal of Bifurcation and Chaos in Applied Sciences and Engineering, vol. 20, no. 5, pp. 1379-1390, 2010.

[8] F. Liang, M. Han, and V. G. Romanovski, "Bifurcation of limit cycles by perturbing a piecewise linear Hamiltonian system with a homoclinic loop," Nonlinear Analysis. Theory, Methods \& Applications, vol. 75, no. 11, pp. 4355-4374, 2012.

[9] F. Liang and M. Han, "Limit cycles near generalized homoclinic and double homoclinic loops in piecewise smooth systems," Chaos, Solitons \& Fractals, vol. 45, no. 4, pp. 454-464, 2012.

[10] Y. Xiong and M. Han, "Hopf bifurcation of limit cycles in discontinuous Liénard systems," Abstract and Applied Analysis, vol. 2012, Article ID 690453, 27 pages, 2012. 


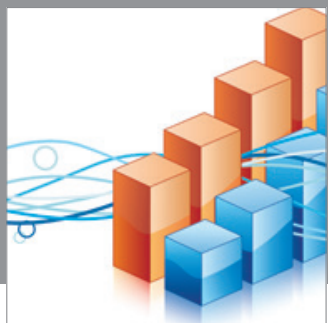

Advances in

Operations Research

mansans

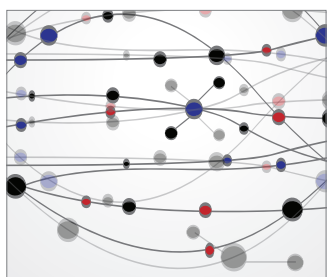

The Scientific World Journal
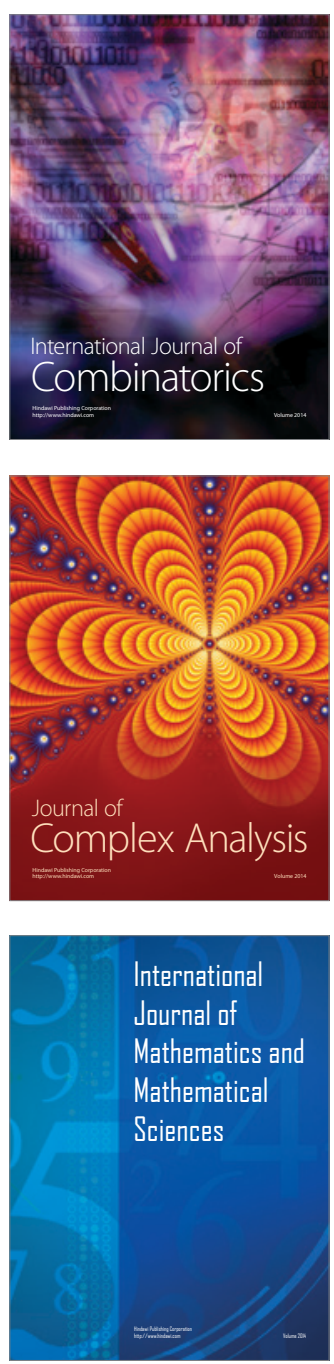
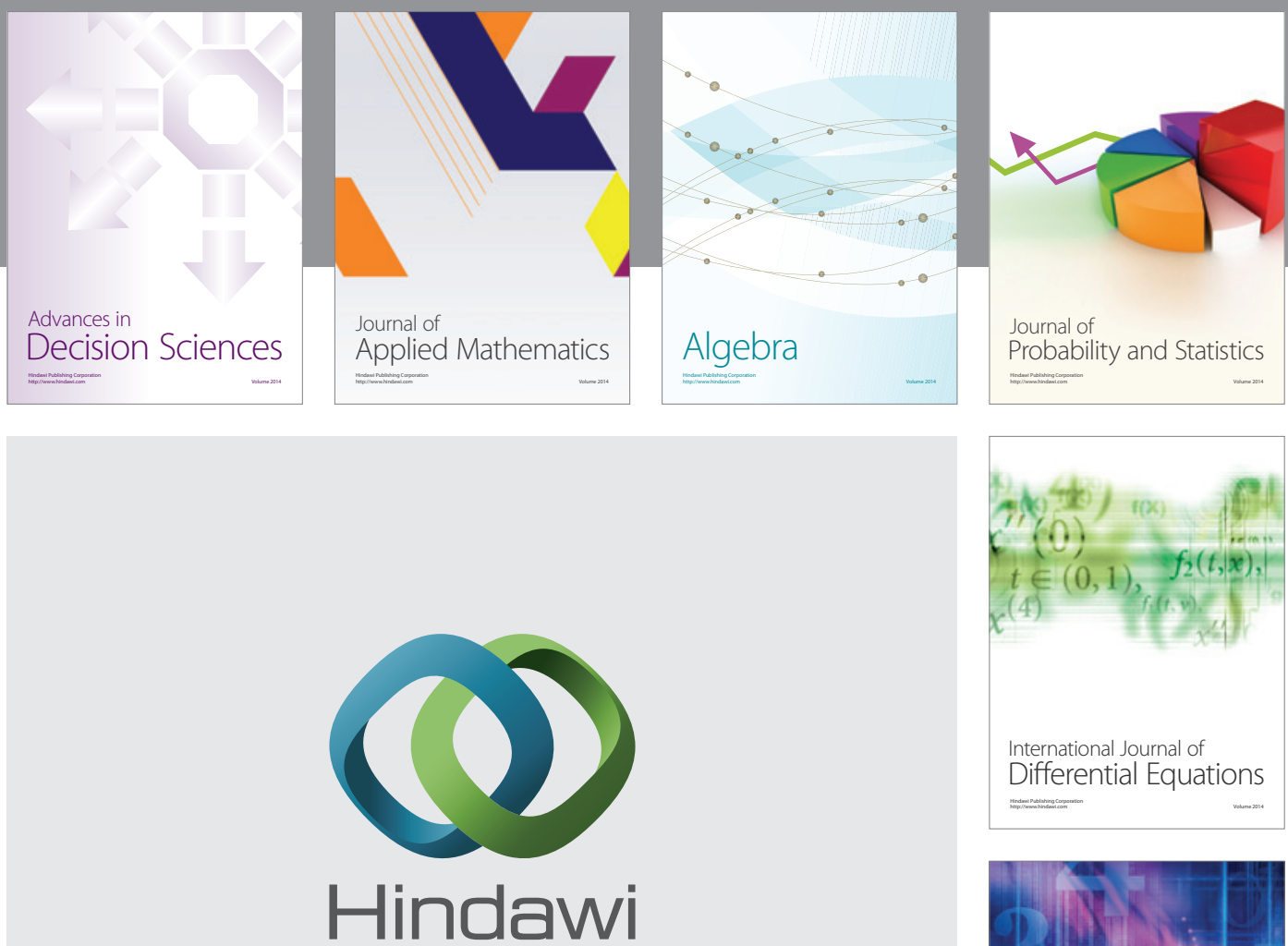

Submit your manuscripts at http://www.hindawi.com
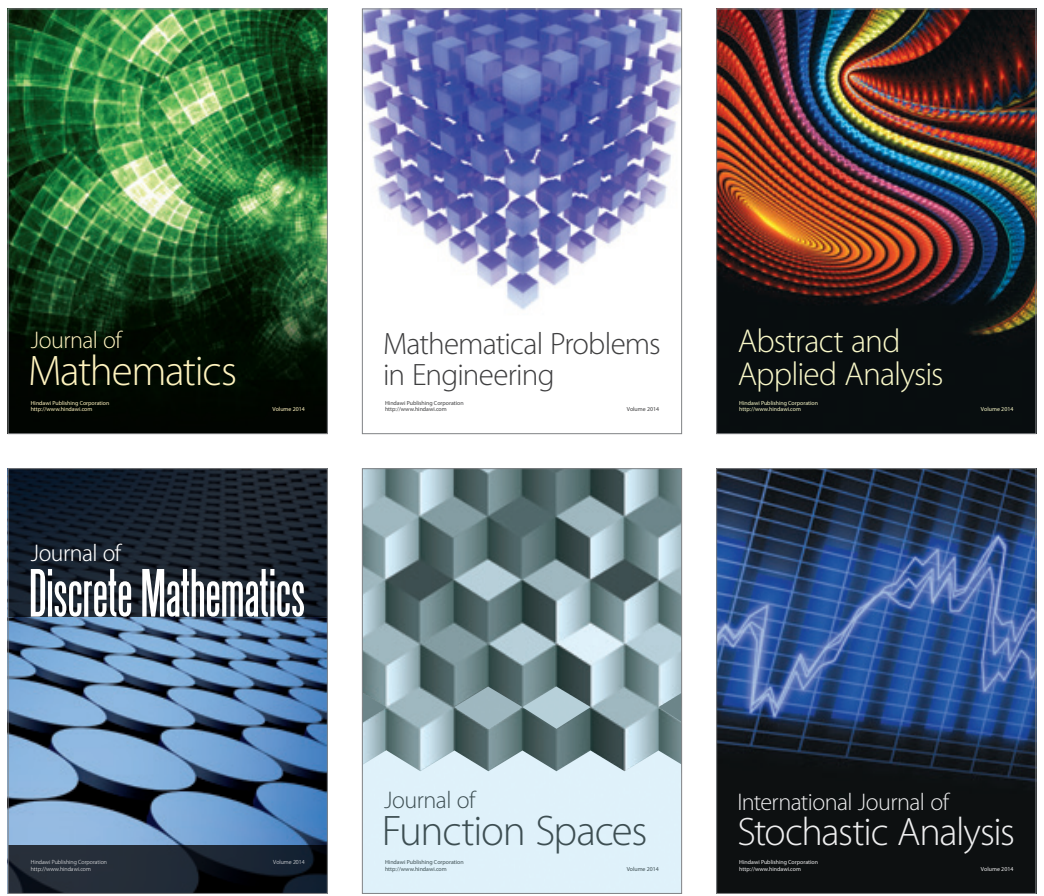

Journal of

Function Spaces

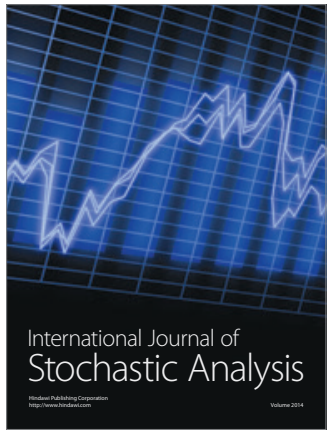

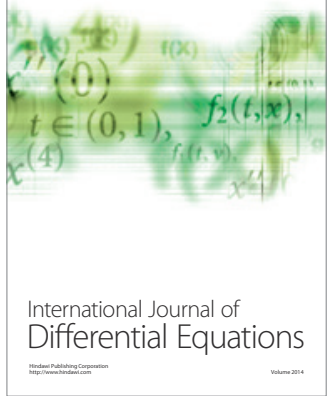
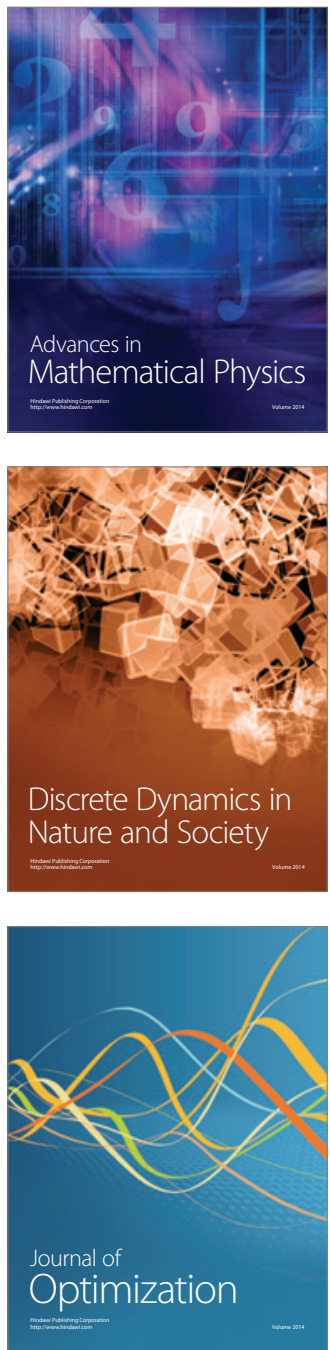